\title{
Probability distributions for maximum wave and crest heights
}

\author{
Prevosto Marc ${ }^{1,}{ }^{*}$, Krogstad Harald $E^{2}$, Robin Agnès ${ }^{1}$
}

1 IFREMER, COM,Ctr Brest, GO, DITI, F-29280 Plouzane, France.

2 NTNU, Dept Math Sci, Trondheim, Norway.

*Corresponding author : Marc Prevosto, email address : marc.prevosto@ifremer.fr

\begin{abstract}
:
The paper discusses short- and long-term probability models of ocean waves. The Gaussian theory is reviewed, and nonlinear short-term probability distributions are derived from a narrow band second-order model. The nonlinearity has different impact on different measurement techniques, and this is further demonstrated for wave data from the WAVEMOD Crete measurement campaign and laser data from the North Sea. Finally, we give some examples on how the short-term statistics may be used to estimate the probability distributions for the maximum waves during individual storms as well as in a wave climate described by long-term distributions. (C) 2000 Elsevier Science B.V. All rights reserved.
\end{abstract}

Keywords : maximum wave height, maximum crest height, non linear models, probability distribution, narrow band model 


\section{INTRODUCTION}

Knowledge of the probability distributions for extreme wave and crest heights is of central importance for offshore and coastal engineering. In the present paper we first outline a general methodology for estimating extreme wave and crest heights in deep and shallow waters based on a probabilistic combination of short and long term wave statistics. Whereas long term wave statistics in general is quite site specific, the short term statistics appears to be rather universal. It is therefore of interest to establish suitable forms and parametrizations of the short term statistics for broader applications.

In the present context the necessary short term wave statistics consists of the probability distributions for the maximum wave and crest heights in a constant sea state. Although it is common, and may be quite adequate, to derive these distributions as the distributions of individual wave or crest heights 
raised to the number of waves occurring during the sea state, this will not be assumed a priori. Since the number of waves occurring during a period of, say 1 hour, may be several hundreds, it would in any case be the very upper tail of the individual wave distribution that is of interest and not its main part.

Gaussian linear wave theory is an important first order approximation, and it is shown by computer simulations that the asymptotic relations for the maximum crest height fit quite well for reasonable wave spectra and durations of the order of 1hour. For the maximum wave height, where simple theoretical expressions are harder to obtain, the simulation results turn out quite close to relations initially derived for narrow-banded spectra by M. Longuet-Higgins [24] and A. Næss [26]. We also discuss how nonlinearities modify the probability distributions, including analytic s (a Rayleigh-Stokes model [25]) and empirical models like the Jahns-Wheeler [15] shallow water model. The non-linear models are applied to analyse the performance of various recording systems where it is shown that instruments like downlooking radars and lasers, buoy and pressure gauges all produce different results for the crest height.

Several data sets in addition to the WAVEMOD data [10] have been available for analysis. We start by summarizing a study carried out for the Norwegian Petroleum Directorate that included several different instruments around the Ekofisk area in the North Sea. The water depth at Ekofisk is about $70 \mathrm{~m}$. The data from Vøringplatået and Haltenbanken in the Norwegian Sea reported next are obtained by large oceanographic data buoys in approximately $1600 \mathrm{~m}$ and $230 \mathrm{~m}$ of water, respectively. The WAVEMOD data analysed below have been collected by two directional Waverider buoys at 10 and 100m water depths and one non-directional Waverider in 20m water depth in the Rethymnon bay north of Crete.

The shallow water data give no support for the Jahns-Wheeler model, but the parametrization which is used was actually derived for quite different conditions. The Rayleigh-Stokes model show reasonable agreement when the Lagrangian character of the buoy and the low frequency filtering in the processing is taken into account. Pure empirical models fitted to the data sets have been based on Weibull probability models. The Weibull distribution is a natural choice, in particular for the maximum wave crest where it is follows from the Gaussian assumption of Linear Wave Theory. For wave height there is no general theoretical foundation for the Weibull form, apart from the above-mentioned narrow-banded models. Narrow-banded models lead in the limit of zero width to Rayleigh distributed wave heights but this is never observed in real wave data.

The measured characteristics of waves like skewness and crest-trough asymmetry are in good agreement with the narrow-band non-linear model, whatever the water depth, and demonstrates clearly the underestimation of horizontal wave asymmetry as compared to buoy measurements. The model also explains why the buoy does not "linearise" the waves equally in deep and shallow water.

\section{PROBABILISTIC MODELS OF OCEAN WAVES}




\subsection{Nested stochastic models}

Ocean wave properties, like individual wave height and period, significant wave and period, and seasonal and long term variations in the wave climate, vary simultaneously on many different time scales. In order to estimate, say the 100 year individual maximum wave height, it is therefore necessary to combine phenomena of highly different time behaviour. It has turned out, see e.g. [2], to be useful to consider the ocean waves as a system of nested stochastic models where model parameters on one time scale become stochastic variables on the next and slower scale. The scales are reasonably well separated, i.e., the individual wave period is $O(10$ seconds), the temporal correlation of the surface elevation at a fixed location $O$ (2minutes) and the duration of a fairly stationary sea state $O(1$ hour). Sea state variations occur on a scale of the order of days, whereas seasonal and climatic scales range from one to many years. In our case, the fastest time scale is the scale associated with individual waves and the slower scales are associated with variations in the sea state.

Let $X(t, s)$ be a stochastic process dependent on time $t$ and a multivariate state variable $s$ varying on a time scale longer than the characteristic time of dependence in $X$ (We assume that this time of dependence is larger, but not very much larger than fast time scale). It is convenient to denote the slow time variation by $\tau$ and consider $s(\tau)$ as a stochastic process in its own right. The fast time $t$ is measured with a time unit $T(s)$ and $X(t, \mathrm{~s})$ is therefore locally stationary over time intervals long compared to $T(s)$ but short compared to variations in $s$.

When deriving extreme value distributions for temporary dependent variables one frequently employs some kind of mixing condition which ensures that maxima occurring in disjoint time intervals are asymptotically independent when the intervals increase. In the present case we shall assume this is the case for intervals that are long with respect to the fast time scale. Such a condition then leads to expressions of the form

$$
P\left(\max _{t} X(t, s) \mid 0 \leq t \leq D\right)=F(x, s)^{D / T(s)}
$$

for fixed $s$ and durations $D$ considerably larger than $T(\mathrm{~s})$. By splitting a time history $s(\tau)$ into segments where the state is (approximately) constant, we obtain by a simple limiting argument that

$$
P\left(\max _{t} X(t, s) \mid 0 \leq t \leq D\right)=\exp \left\{\int_{\tau=0}^{D} \log (F(x, s(\tau))) d \tau / T(s(\tau))\right\}
$$

(See e.g. Borgman [5] or Krogstad[16]). Alternatively, the integral can be written as an integral over the corresponding distribution of states, $\left.\Pi_{e}(s)\right)$,

$$
P\left(\max _{t, s} X(t, s) \mid 0 \leq t \leq D\right)=\exp \left\{\int_{s} \log F(x, s) \Pi_{e}(s) d s / T(s)\right\}
$$


When $D \rightarrow \infty$ and the process $s(\tau)$ is ergodic, $\Pi_{e}(s)$ converges to the stationary distribution $\Pi(s)$ of the slow process. If we further let $1 /\langle T\rangle=\int_{s} \Pi(s) / T(s)$, then

$$
P\left(\max _{t, s} X(t, s) \mid 0 \leq t \leq D\right)=\left|\exp \left\{\int_{s} \log (F(x, s)) \Pi(s)\langle T\rangle d \tau / T(s)\right\}\right|=G(x)^{N}
$$

where $N=D /\langle T\rangle$. Since $G$ has the formal properties of a cumulative probability function, we obtain the familiar form for the maximum of $N$ independent identically distributed events, or since $N$ is typically quite large, the corresponding asymptotic form.

In order to apply Eqn. (4), it is thus necessary to determine (i) the function $F(x, s)$ and (ii) the long term distribution. If one can identify Eqn. (1) by the distribution for the maximum of individual independent events, the choice for $F(x, s)$ is obvious. However, it is important to observe that a form $F(x, s)^{N}$ may well be adequate for reasonably large $N$-s even if $F(x, s)$ is different from the distribution of a single event. In the limit procedure carried out in Eqn. (2), it is tacitly assumed the partition is never finer than that the duration of one section is much longer than $T(\mathrm{~s})$.

A fairly general way of determining $F$ is by means of the Rice formula and the Poisson property of high up-crossings [17]. We recall that the mean up-crossing frequency of a level $x, E\left\{v_{X}{ }^{+}(x)\right\}$, is from the Rice formula given by $a_{X}(x) f_{X}(x)$ where $a_{X}(x)=E\left\{\max (d X / d t(0), 0)\right.$ when $X(0)=x$, and $f_{X}(x)$ is the probability density of $X$. When up-crossings of high levels occur according to a Poisson process, the probability of no up-crossings throughout the interval gives

$$
F(x, s)=\exp \left[-a_{X\left({ }^{\circ}, s\right)}(x) f_{X\left({ }^{\circ}, s\right)}(x) T(s)\right]
$$

As well known, $a_{X}(x)$ is constant for Gaussian processes, and in many cases a slowly varying function of $x$ compared to $f_{X}$.

For ocean waves, the expressions above may be applied in different settings. First of all, they apply to the maximum crest heights in which case $X$ is the surface elevation at a single point and $s$ is the sea state, e.g. varying during a storm as in [5], or given in the form of a long term distributions of significant wave height and mean period as in [16]. However, exactly the same methodology may be applied for $X$ being the significant wave height where $s$ signifies the seasonal and climatic variations for the stochastic process of significant wave height. In the present paper we only consider the first case, also treating the maximum wave height within the same framework. The continuous data recordings from Crete during WAVEMOD reported below made it possible to really check the dependency of maxima in adjacent wave records of duration 30 minutes. As expected, it was not possible to reveal any dependency on a statistically significant level.

A similar approach may also be applied for the maximum period, but instead of the maximum period, 
one is rather interested in the period accompanying the maximum wave height. As long as the sea state is constant, the period distribution for the maximum wave height is simply the conditional period distribution for this particular wave height. The generalization to a varying sea state is discussed in [16].

\subsection{The short term wave statistics}

By the term sea state we shall understand the full condition of the sea defined e.g. in term of the wave spectrum, its derived parameters or whatever is necessary. The most basic parameters defining the sea state are the significant wave height, $H_{S}$, and the mean zero-crossing period, $T_{Z}$. We shall assume that both parameters are defined in terms of the wave spectrum as $H_{s}=4 m_{0}^{1 / 2}=4 \sigma_{0}$ and $T_{z}=\left(m_{2} / m_{0}\right)^{1 / 2}$, where $m_{k}=\int_{0}^{\infty} f^{k} S(f) d f$. The peak period, $T_{p}$, is the period corresponding to the maximum of the spectrum. We shall sometimes apply non-dimensional spectra $S_{0}(x)$ such that

$$
S(f)=\frac{H_{s}^{2} T_{p}}{16} S_{0}\left(f T_{p}\right)
$$

where $\int_{0}^{\infty} S_{0}(x) d x=1$ and $\max _{0}(x)=S_{0}(1)$. The average steepness of the sea is the dimensionless number $s=H_{s} \lambda_{0}$ where $\lambda_{0}$ corresponds to the spectral peak.

Short term wave statistics deals with the properties of individual waves in a constant sea state. The joint distribution for the height and period of individual waves has attracted extensive research, see [6], [22] and [30], and the references therein. However, it is known that the heights of adjacent waves and crests are correlated in time, and the sequence of wave heights is actually sometimes modelled as a Markov chain. Since the function $F(x, s)$ is supposed to define the distribution for the maximum occurring over the duration of the sea state rather than the individual event, it is therefore not necessarily relevant to use the distribution of individual wave or crest heights.

\subsection{Linear and non-linear models for the sea surface}

The first order Gaussian model for the sea surface is based on superposition of unbounded, freely propagating Airy waves fulfilling the dispersion relation. Starting with a Gaussian model as the first approximation, it is possible to compute higher order approximations by perturbation methods.

The most direct effect of the non-linearity is the introduction of a certain skewness in the surface height distribution. It has been shown in [32] that in the uni-directional case, the skewness in deep water is given by $\lambda_{3}=\kappa_{3} / m_{0}^{3 / 2}$ where $\kappa_{3}=3 \int_{0}^{\infty} \int_{0}^{\infty} \min \left(k, k^{\prime}\right) S(f) S\left(f^{\prime}\right) \mathrm{dfd} f^{\prime}$ and $k$ is wavenumber from the dispersion relation. For a spectrum scaled as in Eqn. 6, we obtain $\lambda_{3}=3 \pi s \chi$

$\chi=\int_{0}^{\infty} S_{0}(x)\left[\int_{0}^{x} x^{\prime 2} S_{0}\left(x^{\prime}\right) d x^{\prime}\right] d x$ 
where $s$ is the average steepness. For a narrow spectrum centred at $f_{m}$ we obtain at once $\kappa_{3}=3 k_{m} m_{0}{ }^{2}$ and $\lambda_{3}=3 k_{m} H s / 4=3 \pi s / 2$. A standard JONSWAP spectrum characteristic for wind waves gives about the same value. Analysis of bi-directional spectra in [21] showed that in deep water directionality in general diminished the skewness, which varied between 44 and 101\% compared to the uni-directional value. The exact formula for the skewness in an arbitrary second order random wave field is given in [7].

By assuming a narrow band spectrum centred around $k_{m}$, the first order (Gaussian) wave elevation may be written as a product of an amplitude and a phase time function, $X(t)=\sigma_{0} a(t) \cos (\theta(t))$, where the amplitude and instantaneous frequency are slowly varying. With $\sigma_{0} k_{m}=\pi \mathrm{s} / 2$ as the perturbation parameter, the unidirectional narrow band second order model for the normalized elevation becomes

$$
\zeta(t) / \sigma_{0}=\left(\frac{\pi}{2} s\right) c_{0}(\kappa)+\left(\frac{\pi}{2} s\right) c_{d i f f}(\kappa) a^{2}(t)+a(t) \cos (\theta(t))+\left(\frac{\pi}{2} s\right) c_{s u m}(\kappa) a^{2}(t) \cos (2 \theta(t))
$$

where $\kappa=k_{m} h$ is dimensionless depth. The constants $c_{0}, c_{\text {diff }}$ and $c_{\text {sum }}$ are rather complicated expressions of dimensionless depth, see Appendix 1 [29].The expression for skewness of $\zeta(t)$ is easily seen to be

$$
\lambda_{3}=\frac{3}{2} k_{m} H_{s}\left(c_{\text {diff }}(\kappa)+c_{\text {sum }}(\kappa)\right)=3 \pi s\left(c_{\text {diff }}(\kappa)+c_{\text {sum }}(\kappa)\right)
$$

Actually, it turns out that within the frame of the narrow-banded model, expressions similar to Eqn. 8 are valid whatever property of the waves we consider (free surface elevation, pressure, particle velocities, particle displacements), but the functional form of $c_{0}, c_{\text {diff }}$ and $c_{\text {sum }}$ vary (see Appendix 1) (The coefficients of the narrow band model should not be confused with the coefficients of the regular wave model, as it is made in some papers (e.g. [13]). This equivalence is only valid in infinite depth.

It is interesting to compare the coefficients and the skewness of the narrow band model for the three basic ways of measuring the surface elevation; a fixed point, Eulerian, measurement $\left(\eta_{\text {eul }}\right)$, a particle following, Lagrangian, measurement $\left(\eta_{\text {lag }}\right)$, and a linearly extrapolated pressure measurements $\left(\eta_{p}\right)$. In deep water,

$$
\begin{aligned}
& \eta_{\text {eul }}(t) / \sigma_{0}=a(t) \cos (\theta(t))+\frac{1}{2}\left(\frac{\pi}{2} s\right) a^{2}(t) \cos (2 \theta(t)), \\
& \eta_{\text {lag }}(t) / \sigma_{0}=\frac{1}{2}\left(\frac{\pi}{2} s\right) a^{2}(t)+a(t) \cos (\theta(t)), \\
& \eta_{p}(t) / \sigma_{0}=-\frac{1}{2}\left(\exp 2 \kappa_{z}\right)\left(\frac{\pi}{2} s\right) a^{2}(t)+a(t) \cos (\theta(t)),
\end{aligned}
$$

and in finite depth the relations are stated in Appendix 1.We observe from Eqn. 9 that eulerian and lagrangian measurements will give the same positive skewness, whereas linear extrapolation always give negative skewness. 
In Fig. 1, the $c_{\text {diff }}$ and $c_{\text {sum }}$ coefficients are plotted as functions of the normalised wavelength $\lambda_{m} / h=2 \pi / \kappa$. For small $\lambda_{m} / h$, all the nonlinearity is concentrated in the sum terms (resp. difference terms) when we consider Eulerian (resp. Lagrangian or pressure) measurements. When we go to intermediate water depths both terms participate to the global nonlinearity, but with a value $c_{\text {diff }}{ }^{+} c_{\text {sum }}$ and an offset $c_{0}$ which are the same for Eulerian and Lagrangian measurements. For the pressure measurement, where the coefficients are shown for a measurement point $10 \%$ of the wavelength below the MWL, the extrapolated elevation global coefficient $c_{\text {diff }}{ }^{+} c_{\text {sum }}$ is always negative, as it was for infinite depth, showing the inability of linear extrapolation to furnish good non-linear terms whatever the water depth.

\subsection{Models for the maximum wave and crest heights}

Due to the importance of Gaussian linear wave theory, it is essential to know the form of the $F$-function (Eqn. 1) for this case. Consider a Gaussian ocean surface elevation record $\eta(t), 0<t<T$, taken at a fixed location. The typical ocean wave record has a wave spectrum peaking around $f_{0}=0.1 \mathrm{~Hz}$ and decaying at high frequencies as $f^{-p}$ where $p$ is between 4 and 5 . The corresponding Gaussian process is therefore well-behaved with a correlation extending almost to a few minutes, that is, much shorter that the typical duration of a stationary sea state. The number of up-crossings of a level $x \mathrm{pr}$ time unit is by the Rice formula $\lambda=T_{z}^{-1} \exp \left(-x^{2} /\left(2 m_{0}\right)\right)$. Moreover, high up-crossings tend to occur according to a Poisson point process. Therefore, the probability for the crest height of not exceeding $x$ during the time interval $D$ is

$$
P(\eta \leq x)=\exp (-\lambda D)=\exp \left(-\left(D / T_{z}\right) \exp \left(-x^{2} /\left(2 m_{0}\right)\right)\right) \approx\left(1-e^{-x^{2} /\left(2 m_{0}\right)}\right)^{N}, N=D / T_{z} .
$$

The last approximation is good for high levels when $N>100$ or larger, and is a proper distribution function. Thus, the distribution for the maximum crest height is approximately the same as for the maximum of $N=D / T_{Z}$ independent Rayleigh distributed crest heights. Note also that $N$ is the number of waves rather than the number of crests $\left(D / T_{c}\right)$, which might have been the first choice. In the derivation of Eqn. (13) there is no assumption about narrow-bandedness of the spectrum or the existence of the 4th order spectral moment. Both expressions in Eqn. (13) are of course virtually identical to the asymptotic Gumbel form $\exp \left(-e^{-\left(y-a_{N}\right) / b_{N}}\right), b_{N}^{-1}=a_{N}=(2 \log N)^{1 / 2}, y=x / \sigma_{0}$ when $N$ is large.

There exists no simple expression for the distribution of the height of individual waves in a Gaussian wave record apart from degenerate simple situations. However, very accurate approximations to the distributions of wave height and the simultaneous distributions of height and period have been developed by Lindgren and Rychlik [20] based on so-called Slepian models. These expressions require knowledge of the correlation function (or equivalently the wave spectrum), and lead to rather complicated and nonexplicit expressions for the height distribution. Derivations based on processes with narrow band spec- 
tra are more easily adapted to practical situations, and the works of Longuet-Higgins [22] and Næss [26] are particularly useful. Here it is shown that the distribution of wave height may be expressed by the Weibull model

$P\left(H / m_{0}^{1 / 2} \leq x\right)=\left[1-\exp \left(-\frac{x^{2}}{m_{0}(1-\rho(T / 2))}\right)\right]$

where $\rho$ is the correlation function in the wave record and $T$ is the typical wave period. Actually, the value to use is the minimum of the correlation function, which is typically found to be between -.6 and -.75 for ocean wave spectra (This minimum value depends only on the shape of the spectrum). From Eqn. (14) it is necessary to find the form for the maximum height in a constant sea state, and as noted by Næss [26], applying an assumption of independent wave heights is somewhat inconsistent with the narrow band assumption. Nevertheless, the effect of correlation between the heights of adjacent waves do not appear to have a major effect on the formula when $N$ is large. Eqn. (14) raised to the power $N$ $=D / T_{Z}$. may therefore be a suitable model.

The simplest generalization of the Gaussian model is to assume that the wave record is a deterministic transformation of an underlying Gaussian process, that is,

$\eta(t)=G(X(t))$,

where $G$ is a fixed function. Expressions for $G$ may be determined from observations of the probability distribution of $\eta$, or, as discussed in [30], by fitting the level crossings properties of $\eta$ as obtained from the Rice formula. For the maximum crest height we then have

$P(\max (\eta) \leq x))=\left[1-\exp \left(-g(x)^{2} / 2\right)\right]^{D / T_{z}}$,

where $g$ is the inverse function of $G$. The same argument is not immediately applicable to the maximum wave height since the wave height would be dependent on $G$ both at the crest and at the trough, see also [30]. In fact, it is often noted that the higher crests and shallower troughs seen in real ocean waves tend to balance and give a distribution of wave height closer to the Gaussian result.

The asymptotic extreme value distributions we are encountering are mostly of the Gumbel form, and if $Y$ belongs to the Gumbel class, with an extreme value distribution of the form $\exp \left(-\exp \left(-\left(y-a_{N}\right) / b_{N}\right)\right)$ and $Z=H(Y)$ is a smooth, strictly increasing transformation of $Y$, then $Z$ also belongs to the Gumbel class with asymptotic constants given by $a_{N}=H\left(a_{N}\right), \tilde{b}_{N}=H^{\prime}\left(a_{N}\right) b_{n N}$. This applies in particular for the transformation in Eqn. 15 for which we obtain that the asymptotic constants

$$
a_{N}=G\left(\sigma_{X} \sqrt{2 \log N}\right), b_{N}=G^{\prime}\left(\sigma_{X} \sqrt{2 \log N}\right) /\left(\sigma_{X} \sqrt{2 \log N}\right), N=D / T_{Z} .
$$


We recall that the mode, that is the most probable value of the Gumbel distribution is $a_{N}$, the mean is $a_{N}+.0 .5772 b_{N}$ and the standard deviation $1.28 b_{N}$.

The Jahns-Wheeler model. The Jahns-Wheeler model [15] of crest heights is an empirically based modification of the Gaussian/Rayleigh model to shallow water. The model accounts for an elevated crest height at moderate depths followed by a decrease in very shallow water, governed by the wave amplitude to depth ratio. By introducing dimensionless variables $X=4 \eta_{c} / H_{s}$ and $a=H_{s} / 4 h$ where $\eta_{c}$ is the crest height and $h$ the water depth, the distribution may be expressed, in the same form as in Eqn. (16), as

$$
P(X \leq x)=F_{a}(x)=1-\exp \left[-Y(a x) /\left(2 a^{2}\right)\right]
$$

where $Y(y)=y^{2}\left[1-b_{1} y\left(b_{2}-y\right)\right]$. The values $b_{1}=4.37$ and $b_{2}=0.57$ were obtained by Haring and Heideman [11] using data from the Mexican Gulf. The Rayleigh distribution is recovered for $a=0$ and the function $Y$ is strictly increasing for all positive values of $y$. It is also easily seen that $F_{0}(x)>F_{a}(x), 0<x<b_{2} / a$, whereas the opposite is true when $x>b_{2} / a$. Thus, the probability for the crest of reaching a certain level $x$ is larger than for the Rayleigh model as long as $x<b_{2} / a$ or the level is less than $0,57 \times h$. The obvious expression for the maximum crest height for the Jahns-Wheeler model is, according to the Gaussian limit case,

$$
P\left(X_{\max } \leq x \mid 0 \leq t \leq T\right)=\left(1-\exp \left[-Y(a x) /\left(2 a^{2}\right)\right]\right)^{D / T_{z}}
$$

The asymptotic extreme value distribution is of the Gumbel type, and applying the general result above, we find the constants

$$
\begin{aligned}
a_{N} & =\frac{Y^{-1}\left(2 a^{2} \log N\right)}{a}, \\
b_{N}^{-1} & =\frac{Y^{\prime}\left(a b_{N}\right)}{2 a},
\end{aligned}
$$

where $Y^{-1}$ is the inverse function of $Y$.

The Rayleigh-Stokes model. If we return to Eqn. 8, the amplitude $a(t)$ will be slowly varying compared to $\theta(t)$. Thus, the maximum of $\eta(t)$ will occur around the maximum of $a(t)$ when $\theta(t)$ equal 0 , and hence

$$
\eta\left(t_{c}\right) / \sigma_{0}=\left(\frac{\pi}{2} s\right) c_{0}(\kappa)+a\left(t_{c}\right)+\left(\frac{\pi}{2} s\right)\left(c_{\text {diff }}(\kappa)+c_{\text {sum }}(\kappa)\right) a^{2}\left(t_{c}\right),
$$

where $t_{c}$ are the times of the maxima of the first order Gaussian process $X(t)=a(t) \cos (\theta(t))$. Thus, 
for the maximum we may as well consider the maximum of

$$
Z=\left(\frac{\pi}{2} s\right) c_{0}(\kappa)+X+\left(\frac{\pi}{2} s\right)\left(c_{\text {diff }}(\kappa)+c_{\text {sum }}(\kappa)\right) X^{2}=H(X)
$$

which again is of the transformed Gaussian form. Assuming the expression above for the maximum of $X$ we obtain the Rayleigh-Stokes model:

$P\left(\eta_{\max } \leq x \mid 0 \leq t \leq D\right)=\left(1-\exp \left(-\left(\frac{h\left(x / \sigma_{0}\right)^{2}}{2}\right)\right)\right)^{D / T_{z}}, h(z)=\frac{\sqrt{1+4 \gamma\left(z-\pi s c_{0} / 2\right)}-1}{2 \gamma}$,

where $\gamma=\pi s\left(c_{\text {diff }}(\kappa)+c_{\text {sum }}(\kappa)\right) / 2$. Note that $\operatorname{std}(\eta)=\sigma_{0}+\mathrm{O}\left(s^{2}\right)$ and the asymptotic Gumbel constants for the maximum of $\eta(t)$ are

$$
\begin{aligned}
& a_{N} / \sigma_{0}=\sqrt{2 \log N}+\frac{\pi s}{2}\left[-2 c_{\text {diff }}(\kappa)+(2 \log N)\left(c_{\text {diff }}(\kappa)+c_{\text {sum }}(\kappa)\right)\right] \\
& b_{N} / \sigma_{0}=\left[1+2 \frac{\pi s}{2}\left(c_{\text {diff }}(\kappa)+c_{\text {sum }}(\kappa)\right) \sqrt{2 \log N}\right] /(\sqrt{2 \log N}) \\
& \text { where again } N=T / T_{z} .
\end{aligned}
$$

The Rayleigh-Stokes model has been studied and validated on deep water measurements in [25]. As $c_{0}(\kappa)$ and $c_{\text {diff }}(\kappa)+c_{\text {sum }}(\kappa)$ are the same for Eulerian and Lagrangian measurements, the maximum crest heights given by this second order model will be also the same.

We illustrate the evolution of the distribution of the maximum crest height when propagating from offshore to shallow water depth by two examples. First, the propagation of a wind sea (Fig. 3, top) with off-shore sea state $H_{\mathrm{s}}=5 \mathrm{~m}, T_{\mathrm{m}}=6.3 \mathrm{~s}, \lambda_{\mathrm{m}}=63 \mathrm{~m}$ and $s=0.08$, where the period and the steepness $\mathrm{s}$ are assumed to be constant with the water depth. By limiting the value of $s$, we are in a way taking into account wave breaking. The crest height distributions are quite different from those given by the JahnsWheeler model. This is due to the fact that Jahns-Wheeler model takes steepness into account only by the combination $a=\pi \mathrm{s} /(\mathrm{\kappa} 2)$ whereas it is in this case the main factor of nonlinearity. The second example is the propagation of a swell (Fig. 3, bottom) with offshore sea state: $H_{\mathrm{s}}=2 \mathrm{~m}, T_{\mathrm{m}}=14.2 \mathrm{~s}, \lambda_{\mathrm{m}}=314 \mathrm{~m}$, and $s=0.006$ where the period and $H_{\mathrm{s}}$ are taken as constant with the water depth. Here the bottom effect is the main factor of nonlinearity and the distributions are not very different from Jahns-Wheeler model.

The Jahns-Wheeler and Rayleigh-Stokes models are actually quite incompatible. This is most easily revealed by a Taylor expansion in the steepness of the Rayleigh-Stokes exponent. Neglecting the small and constant shift term $\pi s c_{0}((\kappa) / 2)$, and writing $\gamma=\pi s\left(c_{\text {diff }}(\kappa)+c_{\text {sum }}(\kappa)\right) / 2$ as above, we easily obtain from Eqn.24 $h^{2}(z)=z^{2}\left(1-2 \gamma z+5 \gamma^{2} z^{2}\right)+O\left(\gamma^{3}\right)$. To second order in $\gamma$ this is a similar form as the Jahns-Wheeler exponent, and equating the coefficients of the polynomial, we obtain $b_{1}=5\left(c_{\text {diff }}(\kappa)+c_{\text {sum }}(\kappa)\right)^{2} \kappa^{2}$ and $b_{2}=(2 / 5)\left(\kappa\left(c_{\text {diff }}(\kappa)+c_{\text {sum }}(\kappa)\right)\right)^{-1}$. There seems be no way from these expressions to obtain a constant set of parameters as is used in Jahns-Wheeler model. 


\subsection{Instrument effects}

Whereas Eulerian and Lagrangian measurements will in principle give the correct skewness and hence the correct maximum crest heights according to the second order narrow band theory, the extrapolated pressure shows an important bias. In practice, there are however limitations also for the first two measurement principles. We are here essentially thinking of problems due to the recording principle and not difficulties with sea spray for a radar and breaking waves for a buoy.

Free-floating buoy. Consider a free-floating buoy, that is, a buoy which is considered to follow the movement of the free surface particles in the frequency band of interest. Although we have seen above that the skewness of the free surface elevation should be well estimated, this is in fact not true. All existing buoys obtain the displacement by a double integration of an acceleration measurement. This integration has to apply an attenuation of the very lowest frequencies where the signal to noise ratio is bad. This filtering will therefore drastically decrease the contribution from the $\mathrm{c}_{\text {diff-term, most severe }}$ when the spectral bandwidth is small. If we suppose that the high-pass filtering reduces the $\mathrm{c}_{\text {diff }}$ term to 0 , then the measured skewness will be 0 in deep water while some positive skewness is retained for shallower water (Fig. 1, top-right). This ‘linearization' of the wave profile is actually a well known characteristics of buoy measurements.

Pressure sensor. The signal-to-noise ratio problem with pressure gauges is in a sense similar to the buoy case. The pressure fluctuations down the vertical diminish as $\cosh (k(h-z)) / \cosh (k h)$, and so the variations at high wavenumbers are very small. This requires a high frequency attenuation in the filtering from pressure to surface elevation, and hence a possible decrease in the $\mathrm{c}_{\text {sum }}$-term. The net effect is to decrease the negative skewness ever further (Fig. 1, bottom-right).

A typical example of the measurement of a crest with different types of instruments is given in Fig. 4. The linear profile corresponds to the first order free surface elevation. The Eulerian measurement could be from a perfect radar or wave gauge, it shows clearly the effect of the second order component to increase and to narrow the crest. The Lagrangian measurement corresponds to a perfect particle-following buoy, and shows also an increase of the crest height, but no significant modification of the shape of the crest. This, in fact, follows from the above discussion since the second order component (in deep water) affects the crest heights by a slowly varying time function $\left(c_{\text {diff }}(\kappa) a^{2}(t)\right)$ for a Lagrangian measurement whereas it affects the Eulerian measurement by a wave-scale varying time function $\left(c_{\text {sum }}(\kappa) a^{2}(t) \cos (2 \theta(t))\right)$. If we look at the effect of applying a filter cut-off frequency of $0.03 \mathrm{~Hz}$ (called filtered Lagrangian), which is typical of the Waverider double-integration processing, we loose the main part of the slowly varying second order component and the crest height is reduced to the height of the first order elevation. Similarly, as mentioned above, the extrapolated elevation from a pressure measurement (here, $10 \mathrm{~m}$ below the MWL) filtered with a lowpass filter with a cut-off frequency of $0.3 \mathrm{~Hz}$ which a typical value for the pressure sensor at this depth, gives a drastically underestimated crest 
height. Similar comments may also be made for the trough height.

\section{DATA ANALYSIS}

\subsection{Procedures}

The rationale for choosing parametric distributions based on the Weibull distribution has been explained above and is easily extended to distributions of the form

$$
F(x)=P(X \leq x)=\left[1-\exp \left(-g(x)^{\alpha} / \beta\right)\right]^{N},
$$

where $\mathrm{g}$ is a fixed and strictly increasing function of $\mathrm{x}$. Any data analysis will basically be concerned with the following two questions: Do the data conform to a given model, and if this is not the case, what is the model fitting the data best? The first question may be answered by applying the KolmogorovSmirnov test. In the test, the normalised variable,

$$
U=\left[1-\exp \left(-g(X)^{\alpha} / \beta\right)\right]^{N},
$$

is tested for being uniform on the interval [0,1]. In practice, the empirical distribution function of $U$, $F_{U}(y)$, is formed and the maximum deviation, $d=\max _{(0<y<1)}\left|F_{U}(y)-y\right|$ is the test statistics.

Since Eqn. (27) may be written as

$$
\log \left(-\log \left(1-F(x)^{1 / N}\right)\right)=\alpha \log (g(x))-\log (\beta) \text {, }
$$

it is always possible to estimate $\alpha$ and $\log (\beta)$ as the parameters of a straight line fit to the transformed empirical distribution function, $\log \left(-\log \left(1-F_{e}(x)^{1 / N}\right)\right)$, vs. $\log (g(x))$. The value of $N$ is also of importance, since the fit of such a line will be limited to a range of $x$-s where $g(x)=O\left((\beta \log (N))^{1 / \alpha}\right)$. Since this range varies slowly for large $N$-s, a typical wave record with $N=O(200)$ is nevertheless sufficient to determine the distribution up to $N=O\left(10^{4}\right)$ when $g(x) \approx x$. One problem with real wave records is that they contain a varying number of waves and that the expected number of waves, which would have been the reasonable number to use, is not known. However, the estimate of $T_{Z}$ computed from the spectrum is a quite stable and, moreover, $1 / T z$ is the expected number of waves pr. time unit in the record for a Gaussian sea. If one now wants to merge records with different $H_{S}$ and $T_{Z}$, for a given set of $\alpha$ and $\beta$, the bias-correcting transformation $Y=F_{0}\left(F^{-1}(X)\right)$ on the non-dimensional variable $4 X_{\max } / H_{s}$ will bring the data towards the common distribution $F_{0}(x)=\left[1-\exp \left(-x^{\alpha} / \beta\right)\right]^{N_{0}}$.

Related to the Kolmogorov test is also the possibility of estimating optimal values of $\alpha$ and $\beta$ by simply minimising the deviation $d$. The resulting Kolmogorov probability is then a goodness-of-fit measure. 
Although there is hardly anything wrong with the method per se, it has turned out finding the minimum is not quite straightforward. The solution is typically poorly defined in a narrow, flat and curved "valley". The minimum may therefore occasionally be found far away from the expected solution. Even if such solutions fit the data well, extrapolation with obtained parameters to highly different values of $N$ may be questionable. A reasonable alternative is to keep the $\alpha$ parameter fixed and only fit $\beta$.

\subsection{Analysis of Simulated Wave Records}

Analysis of simulated wave records has turned out to be a valuable tool for assessing the theory [9]. A limited study of Gaussian, linear records has been carried out using standard wave spectra of the JONSWAP form with mean parameters and a $f^{-4.5}$ decay at high frequencies. One set of simulations used a single peaked spectrum with peak period 10s whereas the other used a mixed spectrum consisting of an equal sum of two JONSWAP spectra with period 8 and $12 \mathrm{~s}$, respectively. In both cases 1000 series of 1024s duration with a sampling frequency of $8 \mathrm{~Hz}$ were generated, using the standard method of simulating complex Gaussian Fourier coefficients according to the spectrum and then obtaining the series by an inverse FFT. Sea state parameters and the maximum crest and wave height were then computed for each series.

As expected, some differences in the results were observed according to whether the sea state parameters, i.e. $H_{S}$ and $T_{Z}$, were estimated from the simulated time series or used exactly as computed from the input spectrum. The results from the simulations were tested by the Kolmogorov test as explained above. With 1000 observations the test is quite sensitive. One important and reassuring conclusion from the study is that there appears to be no reason to reject the expression in Eqn. (13) for the maximum crest height. The values $\alpha=\beta=2$ are therefore the simple choice for the Gaussian sea.

The results for the maximum wave height are presented in Table 1. The Kolmogorov probabilities are shown in parenthesis. Here, there is no universally valid theoretically based distribution to check against apart from the Rayleigh distribution which was rejected with very large margin in all cases. The narrow band modification by Næss was tested next. It was necessary to compute the correlation function from the spectra, and in accordance with previous studies, its minimum value was found to be -.70 for the single peaked spectrum and -.65 for the double peaked spectrum. This corresponds to $\beta=6.8$ and 6.6 , respectively. We observe that for both spectra, when the exact expressions for the sea state parameters are used, there is no reason to reject the Næss model. Moreover, fixing $\alpha$ to 2 gives an optimal (in the sense of the Kolmogorov metric) $\beta$ quite close to $2\left(1-\rho_{\min }\right)$. When estimated sea state parameters are used, the fit is somewhat poorer (the test statistics for wave crests showed a slightly lower probability for $\alpha=\beta=2$ also, but not low enough to be rejected). Nevertheless, as long as $\alpha$ is fixed to 2 , the optimal value of $\beta$ does not change much. When both parameters are free to vary, the optimal fits appear to be closer to the parameters we find when analysing real data. Since the 'correct' values in this case appear to be close to the Næss model, this suggests that one should keep $\alpha$ fixed e.g. to 2 also when analysing 
real data.

Previous unpublished simulation studies have also suggested some support for the empirical parameter set $\alpha=2.125, \beta=8.42$ proposed by G. Forristall [8, 9] based on buoy data from the Mexican Gulf. These values were also tested and, as noted by Longuet-Higgins and Forristall, may also be a possible set.

\subsection{The Ekofisk wave crest study}

As an example on how various instruments measure the maximum crest height, we shall briefly discuss a study of North Sea data carried for out Norwegian Petroleum Directorate (NPD). The subsidence of the Ekofisk floor with correspondingly ever more critical wave conditions has actualized the need for accurate measurements of the wave crest height.

The study only considered quite severe sea states, mostly taken from the WADIC material. The WADIC experiment (Allender et al. [1]) was a major effort to validate directional wave instrumentation, with the by-product that other properties of the recording instruments, e.g. their wave profiling capability could also be studied. The data material for some of the data sets was not large (about 30 records) and the study only considered simple Weibull models of the form

$$
P\left(\eta_{\max } \leq x\right)=\left(1-\exp \left(-\frac{\left(4 x / H_{s}\right)^{\alpha}}{\beta}\right)\right)^{\nu / 1_{z}} \text {. }
$$

The fits to the Weibull models were carried out by minimising the Kolmogorov distance while allowing a limited variation on the slope parameter $\alpha$. The resulting optimal parameters are given in Tab.2. Fig. 5 shows a plot where the mode of the maximum crest height is displayed as a function of the number of waves. The results are seen to differ considerably. In particular, fixed instruments like radars and lasers are definitely different from buoys, even laying on different sides of the Gaussian result. That fixed instruments give crest height above the Gaussian results is in accordance with the Rayleigh-Stokes theory. Buoys should measure closer to the Gaussian result, and there is no theoretical reason why they come out below the Gaussian result. This is probable due to the three dimensional character of real waves and that buoys tend to avoid the highest peaks, steered in part by their mooring.

\subsection{Vøringplatået and Haltenbanken deep water measurements}

The WAVEMOD data reported below from Crete are all from relatively shallow water. However, deep water buoy data recorded by the Norwegian national programs have also been available to the project. Unfortunately, the data files only contain estimated sea state parameter and the maximum wave height for each record. The maximum crest heights have not been stored.

The Vøringplatået deep water measurement have been carried out by the OCEANOR ASA for the Norwegian Petroleum Directorate. The water depth is about $1600 \mathrm{~m}$ and the data set comprises 5927 records 
from 1989-03-30 to 1991-06-30 [3]. The data set therefore represents a truly deep water buoy measurement. The sampling frequency was $1 \mathrm{~Hz}$ and the duration of the records $34.15 \mathrm{~min}$.

Wave measurements in the Haltenbanken area (depth about 250m) have been going on since 1974, but the project has had access to the directional measurements from 1980-1988 (12792 records). The sampling frequency for these data is $1 \mathrm{~Hz}$ and the duration $17.04 \mathrm{~min}$.

Since we expect some variation with wave steepness, and one is primarily interested in the larger sea states, only data where the mean steepness (based on $T_{z}$ and not on $T_{p}$ ) was larger than.045 has been included in the analysis.

The results for the two locations are shown in Tables 3 and 4. A somewhat surprising observation is that even if both stations use similar types of buoys, the results are definitely different.

First of all, we note that both data sets could be fitted with $\alpha=2$ to a satisfactory degree in all cases. However, whereas the Vøringplatået measurements shows optimal $\beta$-values in this case of the order of what we expect from the Gaussian theory, the Haltenbanken measurements show definitively higher $\beta$-values. A certain increase in $\beta$ with significant wave height is obvious in both cases.

The Forristall values fit the uppermost data from Vøringplatået, whereas the Haltenbanken data gives no support for these values. However, one should bear in mind that the test, for the large amount of data in the Haltenbanken case, is very selective and even small offsets in the observed $\alpha$ and $\beta$ from the Forristall values will be rejected by the test.

The optimally fitted $\alpha$ and $\beta$ are also quite different for the two data sets, the Vøringplatået showing a quite clear trend with significant wave height. The Haltenbanken data do not show an obvious trend and give values around what has been reported previously.

It is difficult to explain the differences between these two data sets which have been obtained with similar buoys (WAVESCAN and NORWAVE heave/pitch/roll buoys). It is likely that the difference is due to the buoy/mooring behaviour rather than actual changes in the maximum wave height distributions.

\subsection{Analysis of the Crete WAVEMOD data}

This Mediterranean site was located on the north-western coast of the island of Crete [28], with a relatively long fetch in the Aegean sea and a bottom slope around 2.5\%. Three wave buoys were deployed along a line approximately perpendicular to the coast. Two DATAWELL Directional WAVERIDER were located at 10 and $100 \mathrm{~m}$ metres depth and one non directional DATAWELL WAVERIDER buoy was located at $20 \mathrm{~m}$ depth. The campaign lasted from February 1994 until the end of October the same 
year. Although summer and winter climatology are quite different, severe sea conditions $\left(H_{s}>4 \mathrm{~m}\right)$ near shore occurred at any season. Wave directions at the site are predominantly north west to north east. Details of the data collection procedure are given in Table 5.

Skewness. Fig. 6 gives the skewness of the sea surface elevation for all selected time series for each Waverider buoy. It is compared to the skewness given by the narrow band model (Eqn. 9) and the bold line is the mean of measured skewness with increasing model skewness. Note that the measured values are quite low and seldom exceed 0.2 at 100 and $20 \mathrm{~m}$ and 0.3 at $10 \mathrm{~m}$. A significant skewness is observed for the Waverider at $10 \mathrm{~m}$ depth, whereas at $100 \mathrm{~m}$ depth the sea-state is completely "linearised". This point confirms what has been said previously on the effect of the high pass filtering with the buoys. For a comparison, the same plot is given from measurements by Elf at the Frigg offshore site located in the North Sea at $100 \mathrm{~m}$ water depth. The wave data here were obtained with a radar altimeter [30], which is expected to provide more accurate and reliable measurements of crest heights at a fixed point than is the case for buoy measurements. In this case the measured skewness is in very good agreement with the model.

Maximum crest and trough height. A similar conclusions to the above may also be drawn from the maximum crest-maximum trough couples in each record (Fig. 7), again showing the strong underestimation of crest heights with a buoy in deep water. If we compare the regression coefficient between crest and trough heights from measurement and from the Rayleigh-Stokes model, we observe as for the skewness a very good agreement in the case of radar measurements, but a decreasing-with-depth underestimation of the measured asymmetry with buoy measurements. This is well explained by the evolution of $\mathrm{c}_{\text {diff }}$ and $\mathrm{c}_{\text {sum }}$ terms with the water depth (see the paragraph "Free-floating buoy", page 11). For this comparison, the simulated maximum crest and trough heights have been obtained, on each data base, by 10 random draws of a Rayleigh-Stokes random variable corresponding to each $\left(\mathrm{H}_{\mathrm{s}}, \mathrm{T}_{\mathrm{m}}\right)$ couple. For example it corresponds for the Frigg data base to 200000 draws. The maximum crest and maximum trough on a time series of $20 \mathrm{mn}$ are considered independent.

\subsubsection{Distribution of maximum crest height}

We first consider the Jahns-Wheeler and the Rayleigh-Stokes probability models for the maximum crest height given in Eqns. 18 and 24. In order to check the $s$ and $\kappa$ dependence, the data where split into bins with width.01 for $s$ and 0.3 for $\kappa$ and ranging from. 005 to .055 for $s$ and .15 to 3.15 for $\kappa$ (the uppermost class for $\kappa$ was extended to $\infty$ ). Only classes with more than 25 data have been considered for the test. The $10 \mathrm{~m}$ measurements include classes with $\kappa$ ranging from .9 and upwards, the $20 \mathrm{~m}$ measurements from 1.2 , whereas the $100 \mathrm{~m}$ site has only data with $\kappa=3$ or larger.

The Jahns-Wheeler model with the parameters given in Sec. 2.4 was rejected in almost all cases apart 
from the lowest steepness class where the model is close to the Rayleigh model of a Gaussian sea. Overprediction of the crest height was the reason for the rejection. From the comparisons between the Rayleigh-Stokes and the Jahns-Wheeler models above, and the reasonable fit for the Rayleigh-Stokes model reported below, it seems clear that by introducing depth-varying $b_{1}$ and $b_{2}$ parameters in the model, it should be easy also to fit this model to the data, but this has not been pursued further.

The Rayleigh-Stokes model has been considered for two different cases. In the first case, the $c_{\text {diff }}$-term is set to zero, in accordance to the low frequency filtering applied in the processing. The second case assumes a partial removal of this term such that the $c_{\text {diff }}$-term contributes $20 \%$ and the $c_{\text {sum }}$-term $80 \%$. The test acceptance probability has been set to $5 \%$ when the number of data is less than 500 and 1\% when the number of data is larger than 500, and the result from the tests are shown in Table 7 . The results are not quite satisfactory for the model although they improve in the second case. It is not an obvious systematic variation for the rejections apart from the $10 \mathrm{~m}$ data where low values of $\kappa$ appear to be unfavourable for the model.

A closer inspection into cases where the model fail, seems to attribute those to an excessive amount of records where the maximum crest height is too low. It is due to strong wavebreaking situations (spilling breaking waves) which has been observed on the position of the $10 \mathrm{~m}$ buoy, coming from very shallow water conditions in which the model is no more valid.

There is no simple relation between the exact form of the Jahns-Wheeler and Rayleigh-Stokes models and the simpler Weibull distribution which was used for significant wave height. The simplicity of the distribution and its easy fitting to real data nevertheless makes it an interesting choice. For the crest we have chosen to fix $\alpha$ to 2 and only optimize $\beta$, again using the Kolmogorov test probability as the goodness-of-fit measure. This worked quite satisfactory in all cases. The large variation in the number of data in the various $(s, \kappa)$-classes make the results somewhat unstable, but by merging all measurements and fitting a quadratic surface though the data, the contour plot in Fig. 8 results. The general picture is that as the steepness tends to zero, the $\beta$ value approaches 2 as expected in this limit. In deep water, there is also a certain increase in the $\beta$-value as the steepness increases although the apparent drop in the upper right corner is doubtful. The value increases sharply as the water get shallow and the steepness increases. We recall that for the Weibull model, the mode of the asymptotic extreme value distribution is equal to $(\beta \log (N))^{1 / \alpha}$ such that an increase in $\beta$ actually means an increase in the mode.

The variation with significant wave height for the three measurement locations is shown in Fig.9. The $10 \mathrm{~m}$ location stands out from the two other, which are quite similar for low waves. When the wave height increases, the $100 \mathrm{~m}$ drops below 2.0. This is similar to the buoy data in the Ekofisk study above, and is probably due to the mooring effects and a tendency for a floating buoy to avoid the highest wave peaks. 


\subsubsection{Distribution of maximum wave height}

A full test of the Longuet-Higgins/Næss model has not been carried out on the data. The Forristall parametrizations was checked on each set, similar to the Jahns-Wheeler and Rayleigh-Stokes models above, the test showed that the data did not fit the model as good as expected. Dividing the data into classes of significant wave height, the test rejected the model for 3 out of 8 classes for the $100 \mathrm{~m}$ measurements, 2 out of 7 classes for the $20 \mathrm{~m}$ measurements and 4 out of 7 measurements for the $10 \mathrm{~m}$ class. There was no systematic variation seen in the rejections.

Estimation of the $\beta$ parameter when the $\alpha$ parameter is fixed to 2 worked quite well also in this case with values similar to the expected values from the Longuet-Higgins/Naess model (see Fig.10). There is a consistent drop in the $\beta$-value when the wave height increases (the highest sea state for the $100 \mathrm{~m}$ location has few data).

Finally, a fit of both $\alpha$ and $\beta$ showed that this estimation is quite unstable, at least for the moderately sized data sets we use here. The variations with significant wave height displayed in Fig. 11 are difficult to interpret.

\section{EXTREME STATISTICS BY COMBINED SHORT AND LONG TERM STATISTICS}

In order to see the importance of a proper short term statistics in the estimation of extreme wave and crest height, we illustrate the use of the analysed models on two case studies. The first is a storm from the Frigg data base where time series of all sea state parameters are available. The maximum wave and crest height are also available for all each record. A plot of the time history of significant wave height and mean zero-crossing period is shown in Fig. 12.

Figures (13) and (14) now show the probability distributions for the maximum crest and wave heights for the storm integrating over the time history as given in Eqn. 2. For wave crest, we observe that the Rayleigh-Stokes model and the close to the Laser Weibull parametrizations from Ekofisk give virtually identical results. For this case, the Rayleigh-Stokes parametrization has been computed from an expected wave period of $12.5 \mathrm{~s}$ and a water depth of $100 \mathrm{~m}$ throughout the storm. It is also easy to verify that the mode of the distributions are quite close also by plotting a figure similar to Fig. 5 . This is reassuring and adds confidence to the Rayleigh-Stokes model. The Gaussian parametrizations show lower crest heights.

The expected crest height for the three parametrizations is $13.5 \mathrm{~m}$ for the Laser parametrization, 12.1 for the Gauss and 13.1 for the Rayleigh-Stokes model. The observed maximum crest height for the storm was $13.9 \mathrm{~m}$ which is of course quite realistic considering the extension of the probability density shown 
in Fig. 13.

Similarly, the expected maximum wave height for the Forristall parametrization is $21.9 \mathrm{~m}$ and a Weibull model with $\mathrm{a}=2$ and $\mathrm{b}=6.8$ is $22.4 \mathrm{~m}$. The observed maximum wave height was is this case $20 \mathrm{~m}$, slightly low as compared to the bulk of the distribution.

It is also possible, as stated in Eqn. (4), to integrate over a long term distribution, and a three parameter Weibull distribution for significant wave height, which could be typical for an exposed Norwegian Sea location, has been used as input,

$P\left(H_{s} \leq h\right)=1-\exp \left(-\left(\frac{h-H_{0}}{H_{c}}\right)^{\gamma}\right)$

with $\gamma=1.26, H_{\mathrm{c}}=2.14$ and $H_{0}=0.7 \mathrm{~m}$. The integration needs in addition relation for the mean number of waves pr time unit, and the relation derived for the Haltenbanken area was used for simplicity. This relation is actually reasonable site independent in the Norwegian Sea:

$1 /\langle T\rangle=0,234\left(H_{s}[m]\right)^{-0,37}\left[s^{-1}\right]$.

It could be mentioned that some care must be exercised when carrying out the integration which involved terms of highly different size.The results is shown in Fig. 15 and 16. The relative locations are quite similar to the single storm above, but the distributions are considerably shifted towards higher values. Slight irregularities in the Rayleigh-Stokes distribution is due to preliminary inclusion of the model in the SWAP software described in [16].

\section{CONCLUSIONS}

In the present paper we have studied short term wave statistics for the maximum waves and crest height that occur during a constant sea state. It is demonstrated that the Rayleigh-Stokes model, based a second order uni-directional, narrow-band spectral model, is able to give reasonable result for the overall surface steepness as well as the maximum crest height. The model may also be applied to study the performance of various wave recording systems. Although EUlerian and Lagrangian instruments should in principle measure the same skewness of the surface, the necessary low frequency filtering in the processing of buoy data makes the results from radars and buoys genuinely different.

THe Jahns-Wheeler and the Rayleigh-Stokes are further found to be quite incompatible. Not only do they differ in deep water, but there is actually no support for the constants b1 and b2 parameters used in the Jahns-Wheeler model, unless they are fitted specially for every location and conditions they are applied.

Computer simulations of Gaussian waves support the analytic theory for the maximum crest height and narrow band models for the maximum wave height. However, the simulations reveal that obtaining the 
sea state parameters form the same record as the maximum is registered may lead to some bias in the distribution for the maximum.

FOr the analysis of real data, The simple and well-known Kolmogorov test is advocated both for testing the acceptance of a given model, and also, somewhat more unconventionally, for fitting optimal models.

Analysis of several field data sets are carried out in order to validate the models. It is clear that for wave height, there seems to be good support for probability models similar to the theoretically derived narrow-band Gaussian models by Næss [26]. Weibull distributions for wave height is therefore a reasonable choice, but even if there also are a certain support for the Forristall set of parameters, the simulation study, and also the results from the analyses of the real data suggest that the shape parameter in the Weibull distribution (a) should be kept at 2, also when analysing real data.

For the maximum crest height, the Rayleigh-Stokes model has reasonable theoretical support, and it is quite clear that also the simpler Weibull parametrizations which have been used earlier should take both the wave steepness and the dimensionless depth into account.

Two case studies the short term models are combined with long term variations in the sea state have been included to illustrate the theory.

\section{ACKNOWLEDGMENTS}

This work was partly funded by the Commission of the European Communities, Directorate General for Science, Research and Development under contract $n^{0}$ MAS2-CT920025. The participants in the WAVEMOD project were IST from Portugal, the Laboratoire d'Hydraulique de France, STNMTE, STCPMVN and IFREMER from France, MARTEDEC and the National Technical University of Athens from Greece, SINTEF and OCEANOR ASA from Norway, Delft University of Technology from Netherlands and Programa de Clima Maritimo from Spain.

The authors are grateful to Elf for permission of using Frigg Field data, and OCEANOR ASA for providing the data sets from Haltenbanken, Vøringplatået and the WADIC experiment.

\section{REFERENCES}

[1] Allender, J., Audunson, T., Barstow, S.F., Bjerken, S., Krogstad, H.E., Steinbakke, P.,Vartdal, L., Borgman, L.: The Wadic project: A comprehensive field evaluation of directional wave instrumentation, Ocean Eng. , Vol. 16 (1989) pp. 505-536.

[2] Athanassoulis, G.A., P.D. Vranas And T.S. Soukissian: A New Model for Long-Term Stochastic Analysis and Prediction-Part I: Theoretical Background, Journal of Ship Research, Vol. 36, (1992)No. 1, pp. 1-16.

[3] Barstow, S.F.: Environmental Conditions in the Norwegian Sea at Vøringplatået, OCEANOR 
report no. R92005 (1992) (In Norwegian).

[4] Barstow, S. F., Paillard, M., Guedes Soares, C.: Field Measurements of Coastal Waves and Currents in the WAVEMOD Project, Proc. OCEANS 94 Conf.(1994) Brest, France.

[5] Borgman, L. : Probabilities for the highest wave in a hurricane, Proc. ASCE 1973 (1973) 99, NOWW2.

[6] Cavanie, A., Ezraty, R. , Arhan, M.: A statistical relationshipbetween individual heights and period of storm waves, Proc. BOSS'76 (1976) pp. 354-360

[7] Ding, P.-X.: Study of Second-Order Nonlinear Characteristics of Ocean Waves (I), Science in China, vol. 37, no. 3 (1994) pp 625-633.

[8] Forristall, G: On the distribution of wave heights in a storm. J. Geophys. Res. 1975, Vol. 80 (1978) pp. 2353

[9] Forristall, G. : The Distribution of Measure and Simulated Wve heights as a Function of Spectral Shape, J. Geophys. Res. , Vol. 20, no. C6(1984) pp. 10547 - 10552.

[10]Guedes Soares, C., Krogstad, H. K., Prevosto, M.,: WAVEMOD project: Probabilistic Models for Coastal Site Investigations, Proc. OCEANS 94 OSATES Conference (1994), Brest, France, Sept. 1994, Vol. I, pp. 493-497.

[11]Haring, R. E. and Heideman, J. C. : Gulf of Mexico Rare Wave Return Periods. Offshore Technology Conference, OTC 3230 (1978) pp. 1537-1550.

[12]Huang N. E., Long S. R.: An experimental study of the surface elevation probability distribution and statistics of wind-generated waves, J. Fluid. Mech. , Vol. 101(1980) pp. 179-200.

[13]Huang, N.E., Long, S.R., Tung, C.-C., Yuan, Y., Bliven, L.F.: A non-Gaussian statistical model for surface elevation of nonlinear random wave fields", J. Geophys. Res., vol. 88, no. C12, (1983) pp. 7597-7606.

[14]I.A.H.R: List of sea state parameters, supplement to bulletin No 52 (1986).

[15]Jahns H. O. and Wheeler J. D.: Long-Term Wave Probabilities Based on Hindcasting of Severe Storms, J. Petr.Techn. Aug. (1973) pp. 473-486.

[16]Krogstad, H.E.: Height and period distributions of extreme waves. Applied Ocean Research, 7, No. 3 (1985) pp. 158-165.

[17]Labeyrie, J.: Stationary and Transient States of Random Seas», Marine Structures, 3 (1990) pp. 43-58.

[18]Leadbetter, M.R., Lindgren, G. and Rootzen, H.: Extremes and related properties of random sequences and processes, Springer-Verlag, New York(1983).

[19]Leadbetter, M.R. : Extremes and Exceedence Measures for Continuous Parameter Stationary Processes, in J. Galambos et al. (eds.) Extreme Value Theory and Applications, Kluwer Acad. Press, pp. 371-388 (1994).

[20]Lindgren, G. and I. Rychlik: Slepian models and regression in crossings and extreme value theory, Int. Stat. Reviews, vol. 59 (1991) pp. 195-225.

[21]Longuet-Higgins, M. S.:The effect of non-linearities on statistical distributions in the theory of sea 
waves, J. Fluid. Mech. , Vol. 17 (1963) pp. 459-480.

[22]Longuet-Higgins, M.S.: On the joint distribution of the periods and amplitudes of sea waves, $J$. Geophys. Res., Vol. 80, no. 18(1975) pp. 2688-2694.

[23]Longuet-Higgins, M.S.: Eulerian and Lagrangian aspects of surface waves, J. Fluid. Mech. , Vol. 173(1986) pp. 683-707.

[24]Longuet-Higgins, M.S.: On the distribution of the heights of sea waves: Some effects of nonlinearity and finite band width, J. of Geophys. Res., 85(1980) pp. 1519-1523.

[25]Nerzic, R., Prevosto, M.: A Weibull-Stokes Model for the Distribution of Maximum Wave and Crest Heights, Proc. of the 7th ISOPE Conf., vol. III(1997) pp. 367-377.

[26]Næss, A.: On the distribution of crest to trough wave heights, Ocean Eng., vol. 12, no. 3, (1985) pp. 221-234.

[27]Ochi, M. K., Wang, W.: Non-Gaussian characteristics of coastal waves, Proc. of the 19th Coastal Eng. Conf., Houston(1984) pp. 516-531.

[28]Barstow, S.F., Paillard, M., Guedes Soares, C.: Field Measurements of Coastal Waves and Currents in Portugal and Greece in the WAVEMOD Project, Proc. OCEANS 94 OSATES Conference (1994), Brest, France, Sept. 1994, Vol. I, pp. 487-492.

[29]Prevosto, M., to be published

[30]Robin, A., Olagnon, M.: Occurrence of extreme waves with respect to significant wave height, Proc. of the 10th Int. Conf. on Offshore Mechanics and Arctic Engineering, Vol. 2 (1991) pp. 1-11.

[31]Rychlick, I., P. Johannesson and M.R. Leadbetter: Modelling and Statistical Analysis of Ocean Wave Data Using Transformed Gaussian Processes, Marine Structures, 10, No. 1 (1997) pp.13-48.

[32]Skrokosz, M. A., Longuet-Higgins, M. S.: On the skewness of sea-surface elevation, J. Fluid. Mech., Vol. 164 (1986(pp. 487-497. 
1. Results from the simulation studies. Kolmogorov probabilities shown in parentheses.

\begin{tabular}{|c|c|c|c|c|}
\hline Simulation & $\begin{array}{l}\text { Test of Næss } \\
\text { narrow band model }\end{array}$ & $\begin{array}{l}\text { Test of Forristall values, } \\
\alpha=2.125, \beta=8.42\end{array}$ & $\begin{array}{l}\text { Optimal } \beta \\
\text { for } \alpha=2\end{array}$ & $\begin{array}{l}\text { Optimal } \\
\alpha \text { and } \beta\end{array}$ \\
\hline $\begin{array}{l}\text { Single peaked spectrum, } \\
\text { Exact sea state parameters }\end{array}$ & 2, $6.8(0.98)$ & 0.12 & $\begin{array}{l}6.78 \\
(.98)\end{array}$ & $\begin{array}{l}1.96,6.35 \\
(0.997)\end{array}$ \\
\hline $\begin{array}{c}\text { Single peak spectrum, } \\
\text { Computed sea state pa- } \\
\text { rameters }\end{array}$ & $2,6.8(\sim 0)$ & $\sim 0$ & $\begin{array}{c}6.94 \\
(.005)\end{array}$ & $\begin{array}{l}2.42,14.7 \\
(0.86)\end{array}$ \\
\hline $\begin{array}{l}\text { Double peaked spectrum, } \\
\text { Exact sea state parameters }\end{array}$ & $2,6.6(0.30)$ & .21 & $\begin{array}{c}6.58 \\
(0.40)\end{array}$ & $\begin{array}{c}2.158 .63 \\
(.93)\end{array}$ \\
\hline $\begin{array}{c}\text { Double peaked spectrum, } \\
\text { Computed sea state pa- } \\
\text { rameters }\end{array}$ & 2, $6.6(0.04)$ & .008 & $\begin{array}{c}6.59 \\
(0.06)\end{array}$ & $\begin{array}{l}2.38,13.1 \\
\quad(0.81)\end{array}$ \\
\hline
\end{tabular}

2. Various short term parametrizations for the CDF of maximum crest height for data from the Ekofisk area.

\begin{tabular}{|c|c|c|c|c|c|}
\hline Case & OWNER & Sea states & Size & $\alpha$ & $\beta$ \\
\hline Gaussian sea & - & - & - & 2 & 2 \\
\hline Laser (average) & WADIC group & $>$ & $\sim 57$ & 2 & 2.5 \\
\hline Wavestaff & WADIC group & $8 \mathrm{~m}<\mathrm{Hs}$ & $\sim 30$ & 1.99 & 2.20 \\
\hline Large oceanographic buoy & N/A & $8 \mathrm{~m}<\mathrm{Hs}$ & $\sim 100$ & 2 & 1.85 \\
\hline Pressure cells, extrapolated & NPD/DHI & $9 \mathrm{~m}<\mathrm{Hs}$ & $\sim 30$ & 2.05 & 1.79 \\
\hline
\end{tabular}

3. Results from Haltenbanken. Weibull parametrizations for the maximum wave height for various classes of significant wave height.

\begin{tabular}{|c|c|c|c|c|}
\hline Case & No. of data & $\alpha=2$ & $\begin{array}{c}\text { Test of Forristall's } \\
\text { values, } \alpha=2.13, \beta=8.42\end{array}$ & Optimal $\alpha$ and $\beta$ \\
\hline $2-3 \mathrm{~m}$ & 1534 & $6.72(.34)$ & .09 & $2.148 .730(.999)$ \\
\hline $3-4 \mathrm{~m}$ & 1243 & $6.91(.54)$ & .0 & $2.087 .01(.95) 0$ \\
\hline $4-5 \mathrm{~m}$ & 798 & $7.05(.60)$ & .0 & $2.159 .22(.999) 0$ \\
\hline $5-6 \mathrm{~m}$ & 414 & $6.98(.25)$ & .0001 & $2.3312 .79(.99)$ \\
\hline $6-7 \mathrm{~m}$ & 215 & $7.14(.43)$ & .0 & $2.159 .31(.74)$ \\
\hline $8 \mathrm{~m}<$ & 111 & $7.36(.93)$ & .0 & $2.2010 .61(.998)$ \\
\hline
\end{tabular}


4. Results from Vøringplatået. Weibull parametrizations for the maximum wave height for various class es of significant wave height.

\begin{tabular}{|c|c|c|c|c|}
\hline Case & No. of data & $\alpha=2$ & $\begin{array}{r}\text { Test of Forristall's } \\
\text { values, } \alpha=2.13, \beta=8.42\end{array}$ & Optimal $\alpha$ and $\beta$ \\
\hline $2-3$ & $(670)$ & $6.25(.99)$ & .0 & $1.965 .75(.999)$ \\
\hline $3-4$ & $(610)$ & $6.30(.90)$ & .0 & $1.905 .25(.99)$ \\
\hline $4-5$ & $(377)$ & $6.43(.96)$ & .0 & $1.996 .29(.97)$ \\
\hline $5-6$ & 250 & $6.51(.37)$ & .02 & $2.2710 .82(.85)$ \\
\hline $6-7$ & 145 & $6.77(.81)$ & .66 & $2.179 .25(.96)$ \\
\hline $7-8$ & 72 & $6.50(.80)$ & .40 & $2.2510 .18(.99)$ \\
\hline $8 m<$ & 57 & $6.50(.48)$ & .24 & $2.6521 .02(.99)$ \\
\hline
\end{tabular}

5. Data collection procedures for the WAVEMOD measurements at Crete.

\begin{tabular}{|c|c|c|c|c|c|}
\hline Instrument & $\begin{array}{c}\text { Depth } \\
(\mathrm{m})\end{array}$ & $\begin{array}{c}\text { Sampling freq. } \\
(\mathrm{Hz}) .\end{array}$ & $\begin{array}{c}\text { Rec. duration } \\
(\mathrm{min})\end{array}$ & $\begin{array}{c}\text { Number of valid time } \\
\text { series }(\times 20 \mathrm{mn}) \\
\left(\mathrm{H}_{\mathrm{s}}>0.4 \mathrm{~m}\right)\end{array}$ & $\begin{array}{c}\text { Maximum } \mathrm{H}_{\mathrm{s}} \\
(\mathrm{m})\end{array}$ \\
\hline $\begin{array}{c}\text { DWR } \\
(\varnothing 90 \mathrm{~cm})\end{array}$ & 10 & 1.28 & 20 & 4648 & 4.4 \\
\hline $\begin{array}{c}\text { WR } \\
(\varnothing 70 \mathrm{~cm})\end{array}$ & 20 & 2.56 & 20 & 5013 & 5.0 \\
\hline $\begin{array}{c}\text { DWR } \\
(\varnothing 90 \mathrm{~cm})\end{array}$ & 100 & 1.28 & 20 & 5021 & 5.7 \\
\hline
\end{tabular}

6 . Regression between maximum crest and maximum trough: Crest $=\alpha x$ Trough

\begin{tabular}{|l|c|c|}
\hline \multicolumn{1}{|c|}{ I } & $\begin{array}{c}\alpha \text { coefficient } \\
\text { from measurement }\end{array}$ & $\begin{array}{c}\alpha \text { coefficient } \\
\text { from model }\end{array}$ \\
\hline Buoy 10m - Crete & 1.08 & 1.151 \\
\hline Buoy 20m - Crete & 1.03 & 1.145 \\
\hline Buoy 100m - Crete & 0.99 & 1.173 \\
\hline Radar 100m - Frigg & 1.16 & 1.162 \\
\hline
\end{tabular}


7 . Test of the R/S model for the WAVEMOD Crete measurements.

\begin{tabular}{|c|c|c|c|}
\hline Case & $\begin{array}{c}10 \mathrm{~m} \\
\text { Rejected hyp. }\end{array}$ & $\begin{array}{c}20 \mathrm{~m} \\
\text { Rejected hyp. }\end{array}$ & $\begin{array}{c}100 \mathrm{~m} \\
\text { Rejected hyp. }\end{array}$ \\
\hline Only $\mathrm{c}_{\text {sum }}$-term & 11 of 25 & 8 of 23 & 1 of 5 \\
\hline $20 \% \mathrm{c}_{\text {diff-term }-80 \% \mathrm{c}_{\text {sum }} \text {-term }}$ & 7 of 25 & 4 of 23 & 1 of 5 \\
\hline
\end{tabular}

1 . Second order coefficients versus wavelength/depth ratio

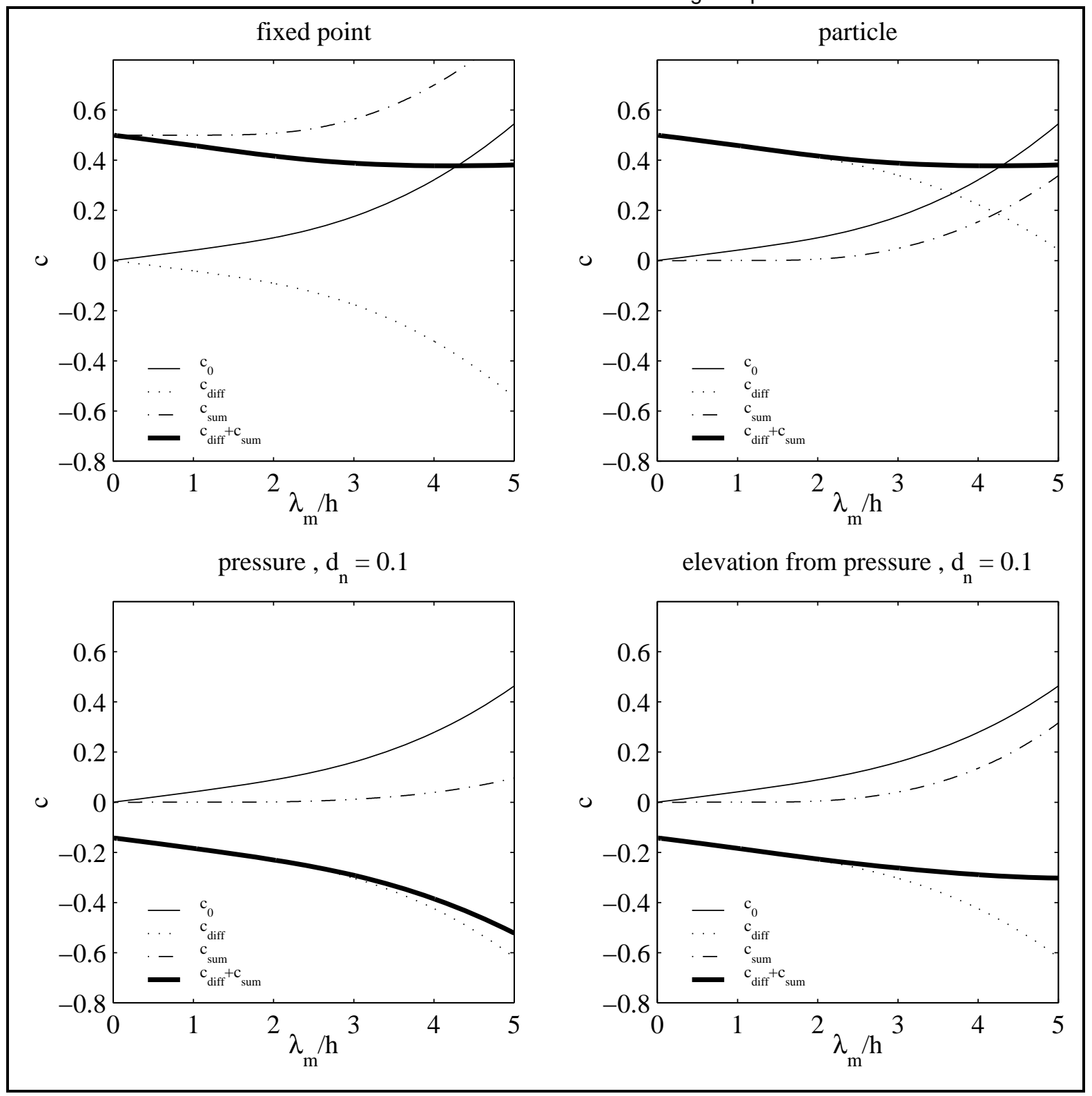


2. Distribution of maximum crest from Jahns-Wheeler model

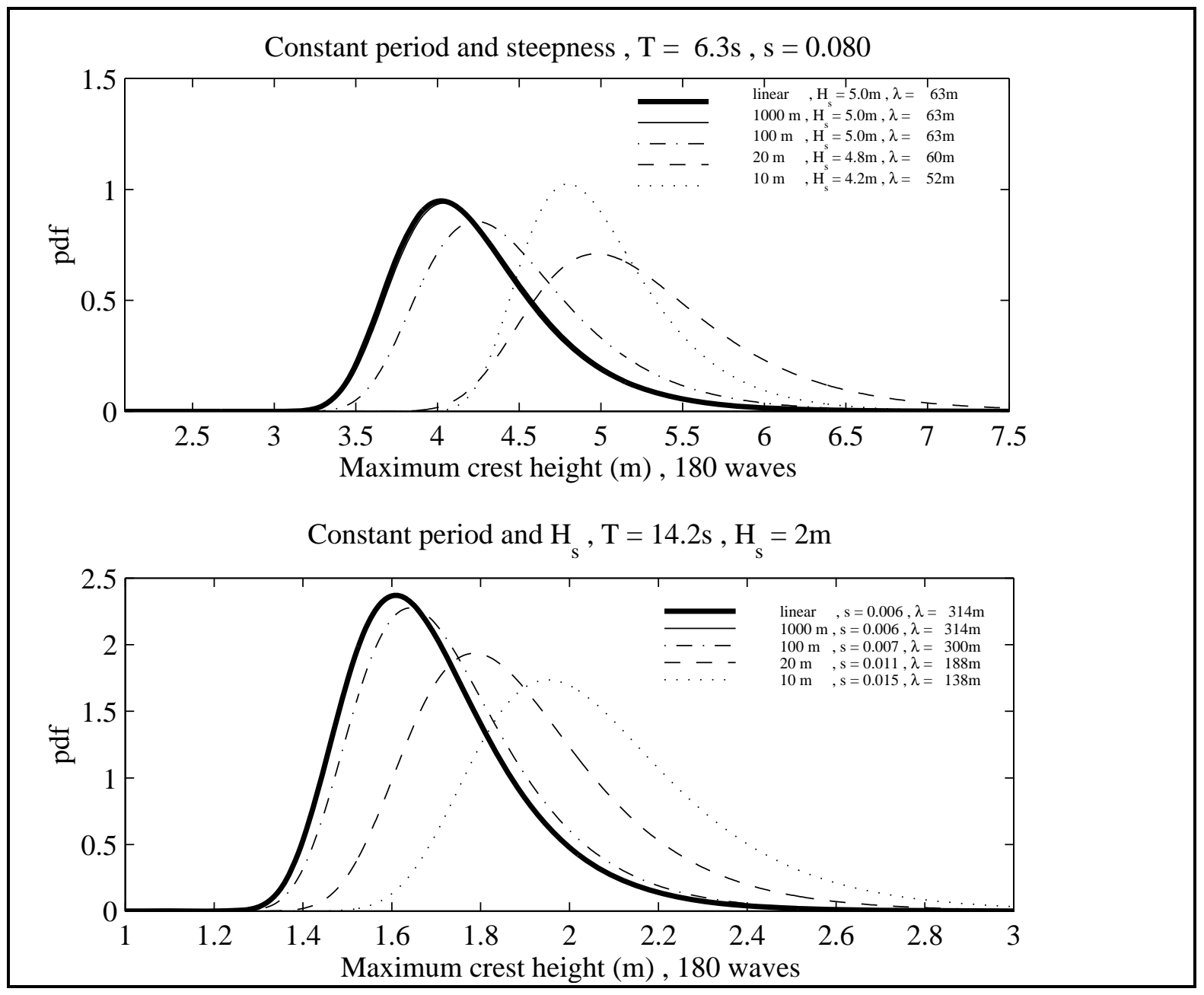


3 . Distribution of maximum crest from Rayleigh-Stokes model

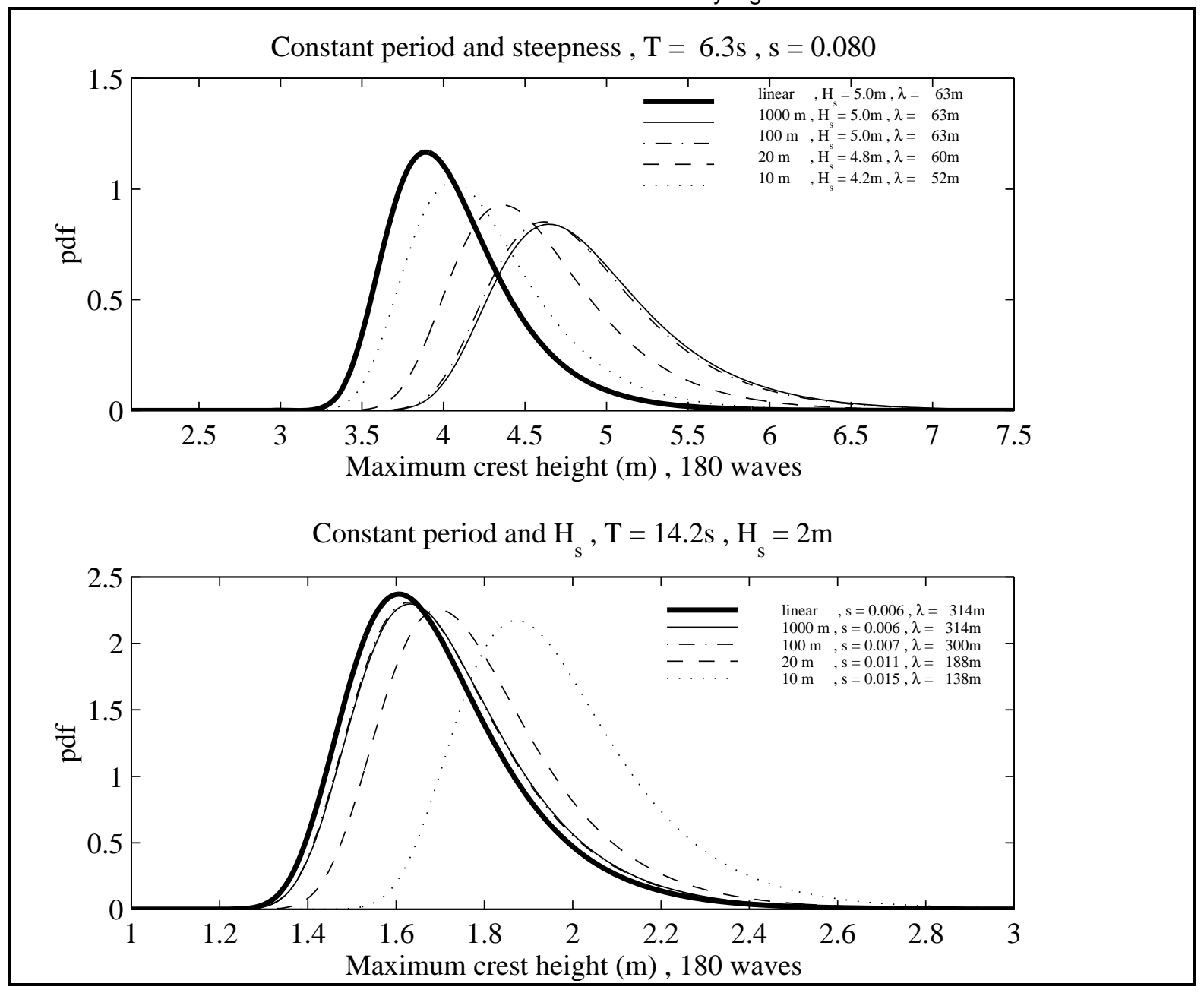

4. Example of crest measurements

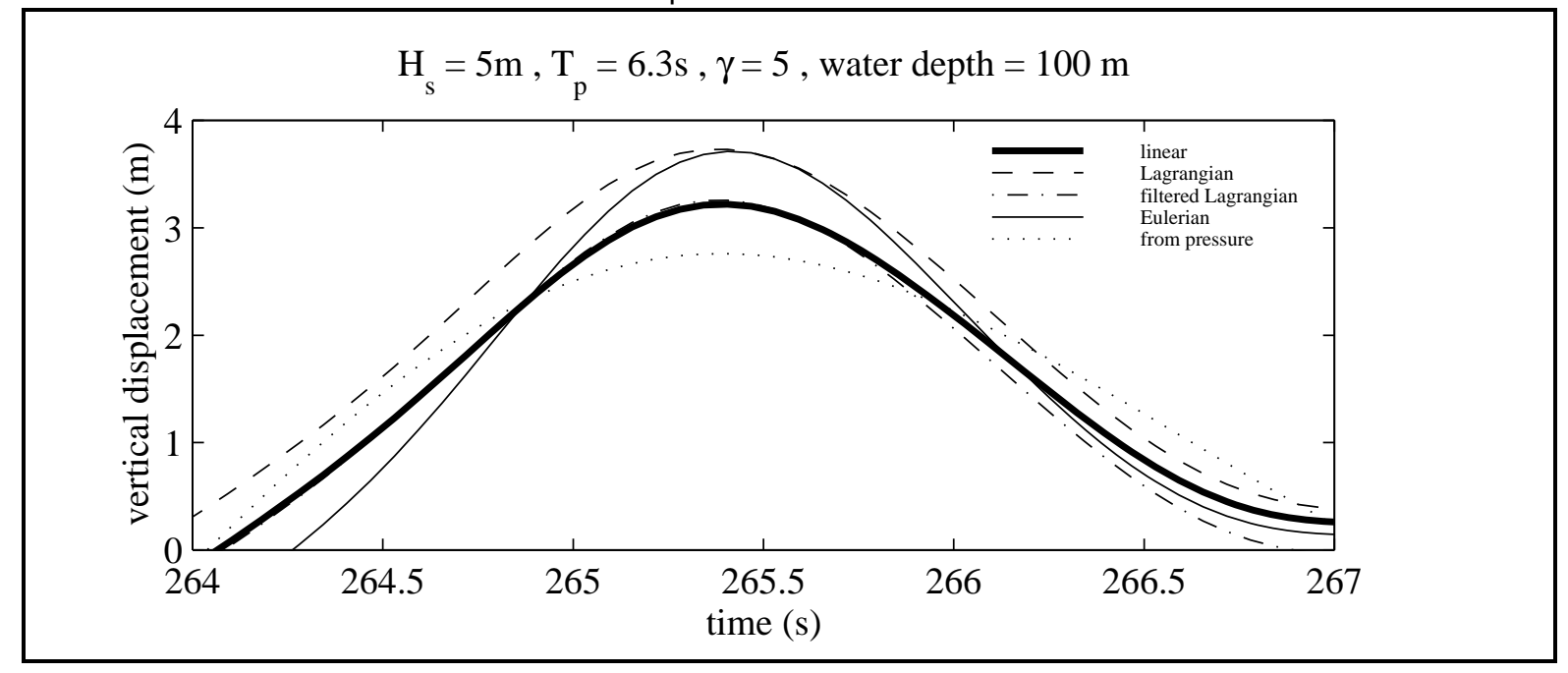


5. Mode (most probable) relative crest height for various instruments from the Ekofisk area: laser(L), wavestaff(S), gaussian sea $(G)$, large oceanographic buoy $(B)$ and inverted pressure recording $(P)$.

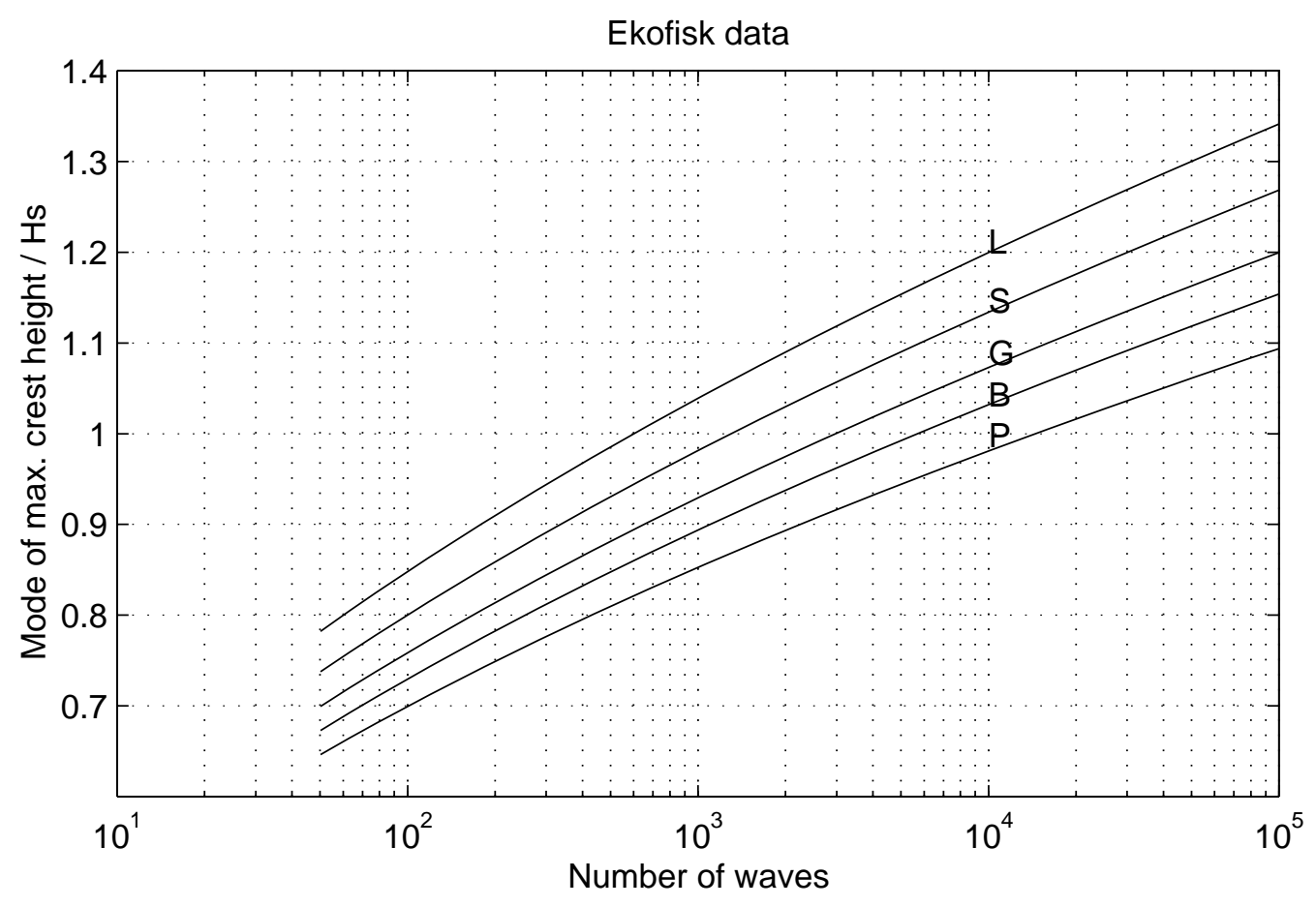


6. Measured skewness versus skewness from marrowbone transfer model

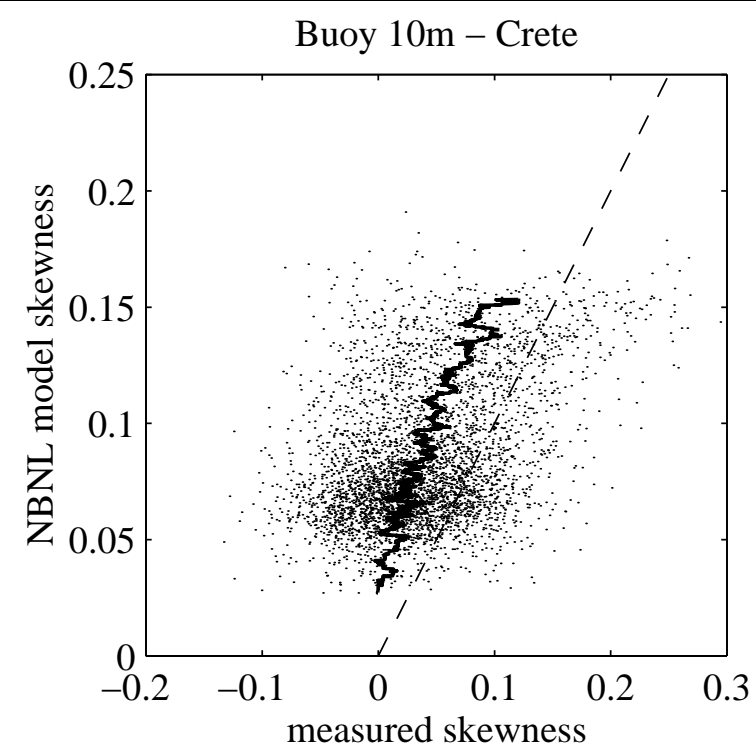

Buoy 20m - Crete
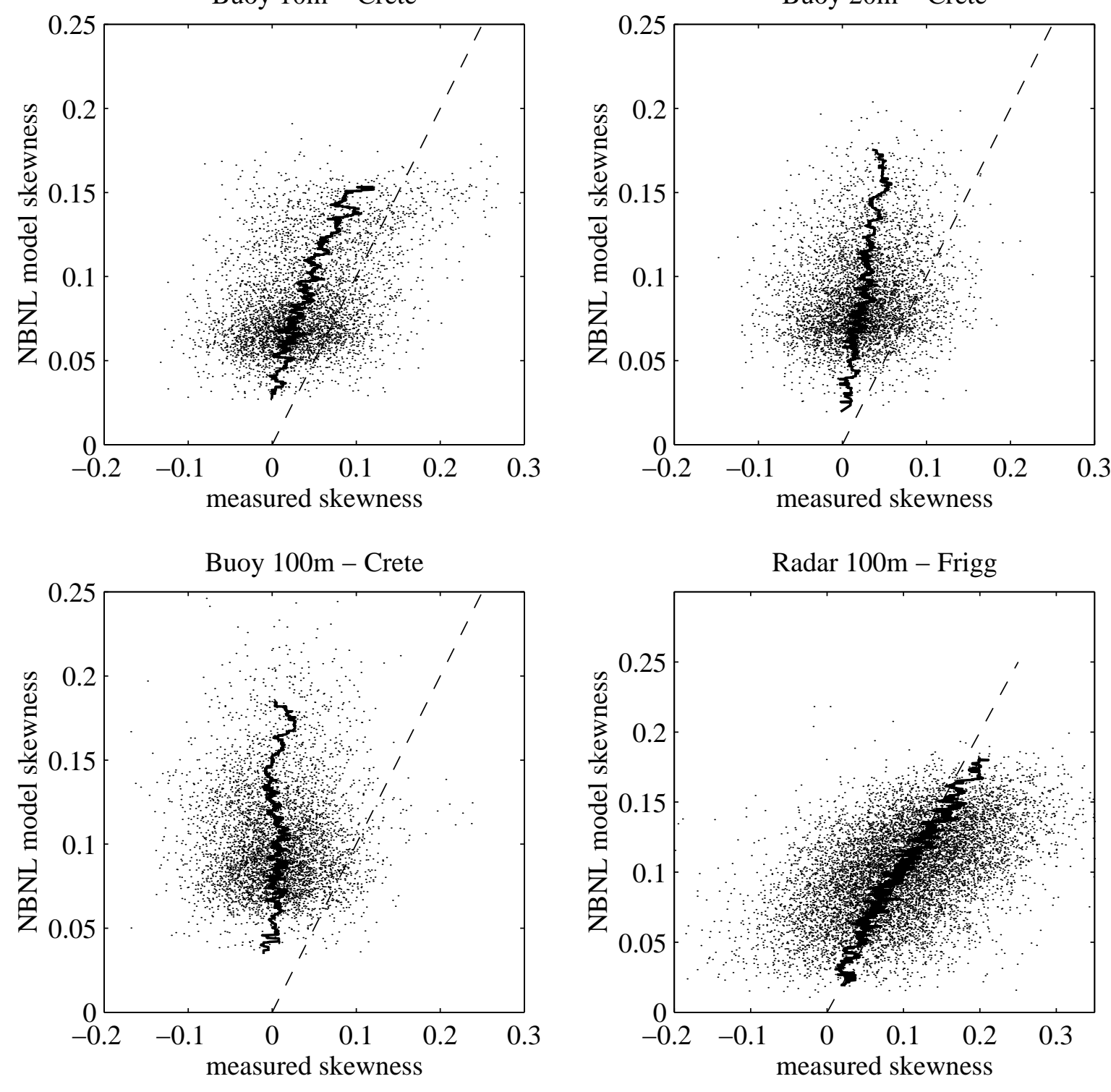
7 . Maximum crest height vs maximum trough height
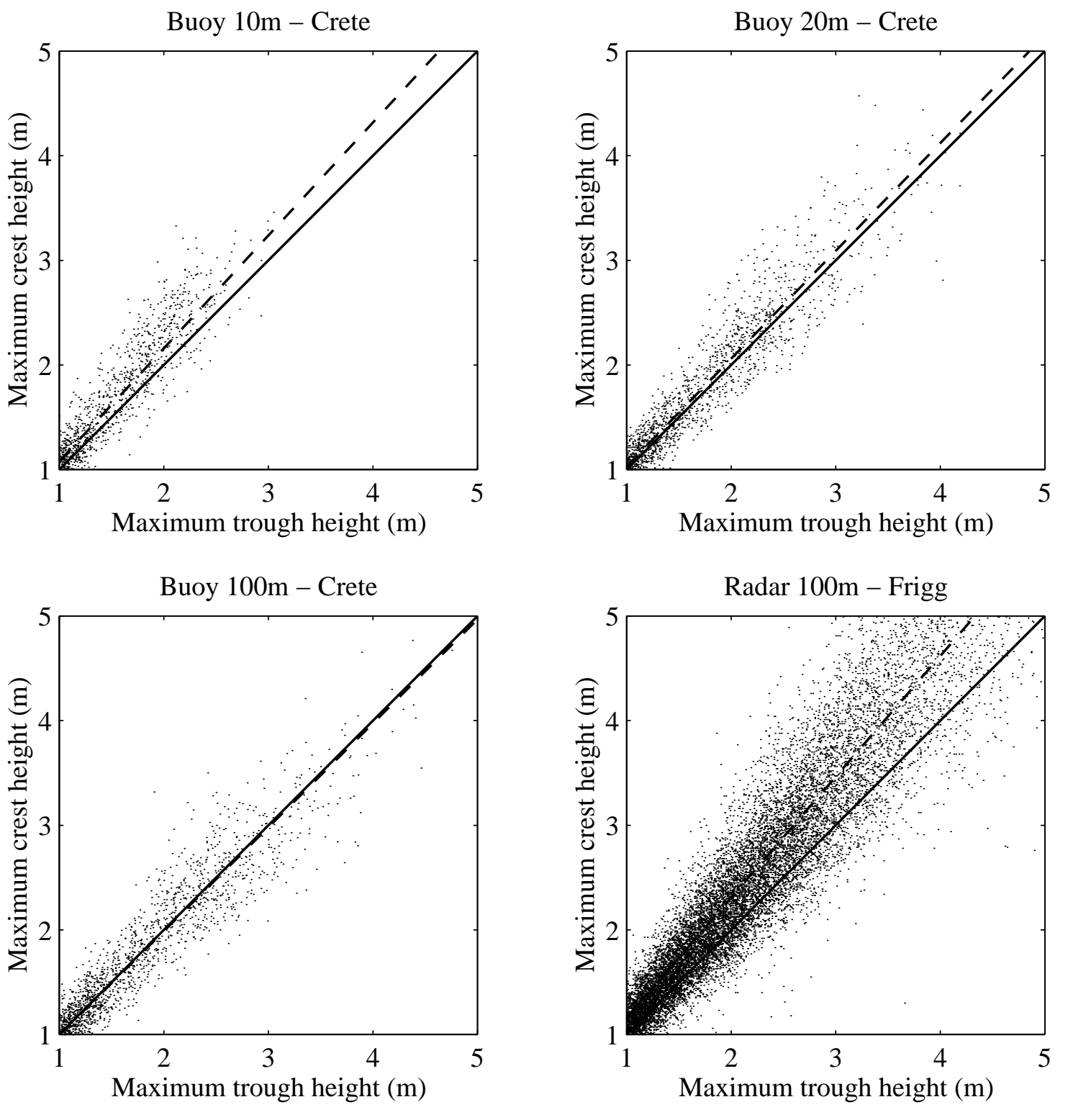
8. Contour plot of $\beta$-parameter as function of $s$ and $\kappa$. Merged data set from all three Crete locations.

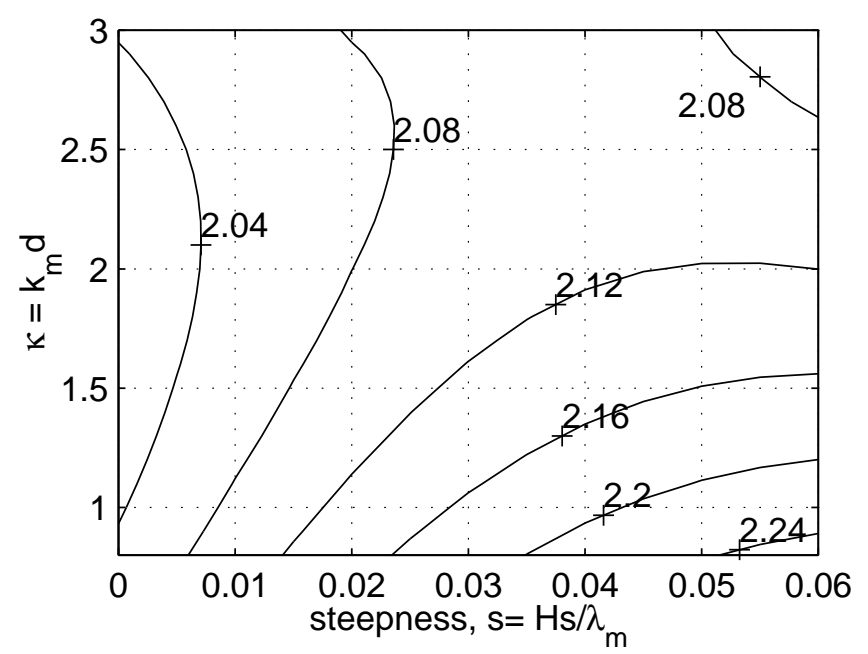

9. Variation of $\beta$-parameter with $\alpha$ fixed to 2 for the maximum crest height when $H_{s}$ varies.

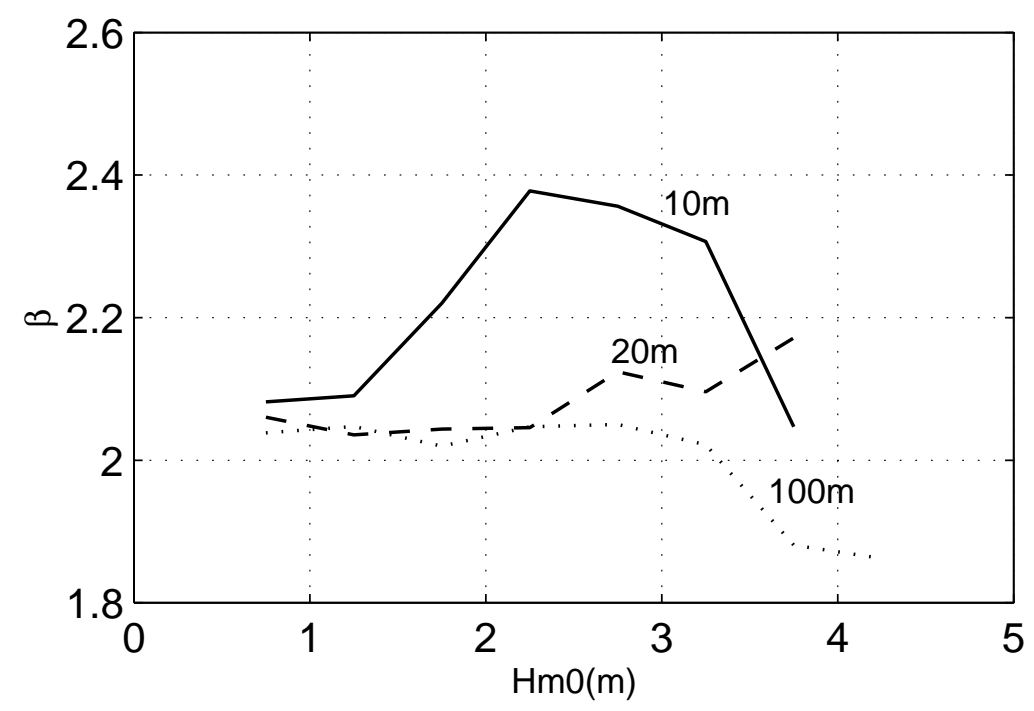


10. Variation of $\beta$-parameter with $\alpha$ fixed to 2 for the maximum wave height when $H_{s}$ varies.

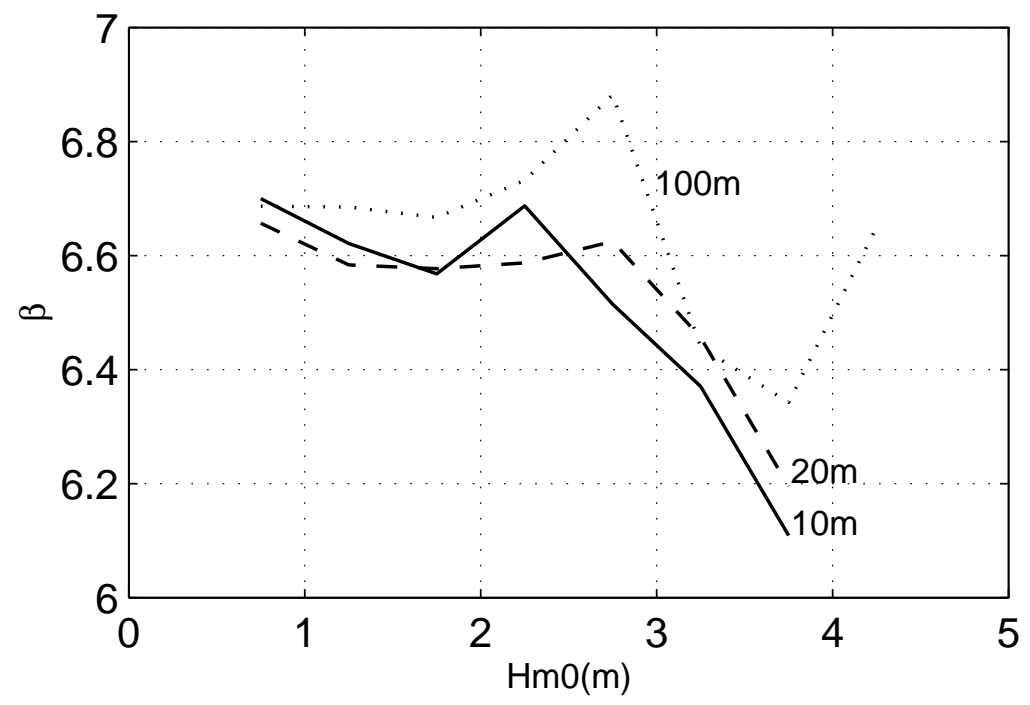


11. Optimal fit of both $\alpha$ and $\beta$ for the maximal wave height as a function of $H_{S}$.
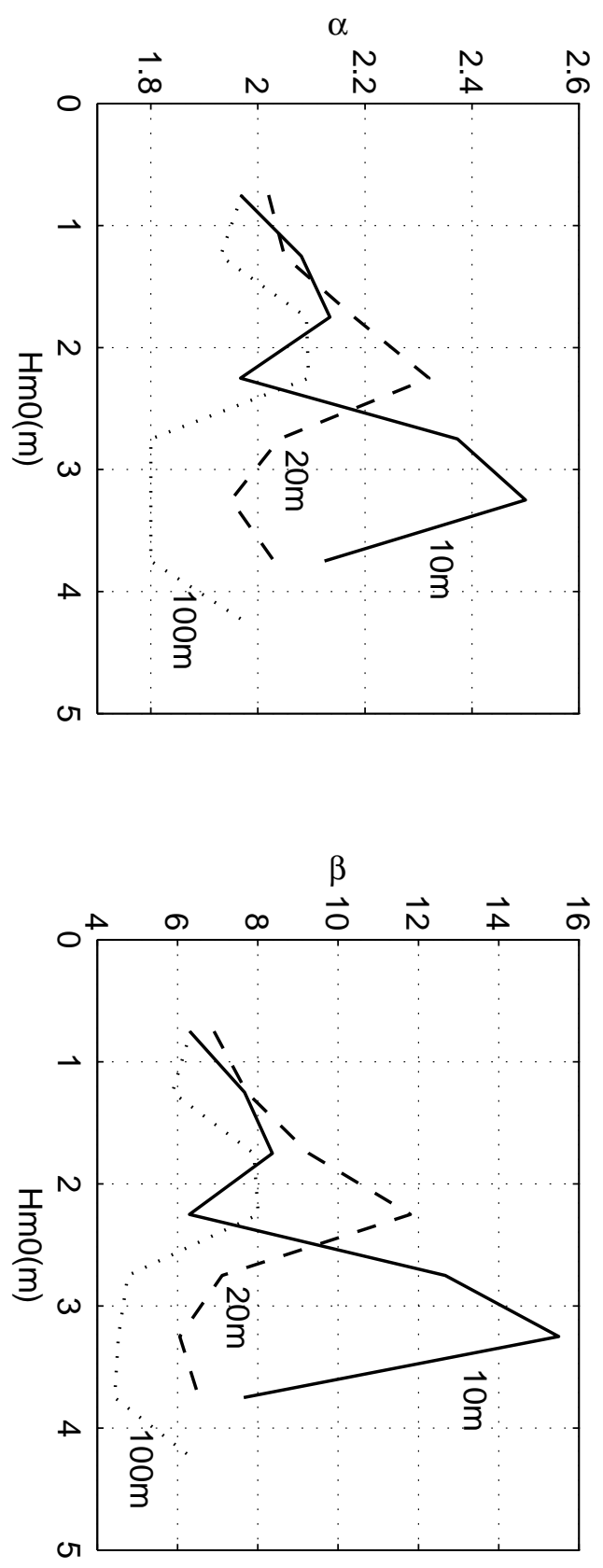
12. Time history of sign. wave height and mean period during the Frigg storm

Frigg storm

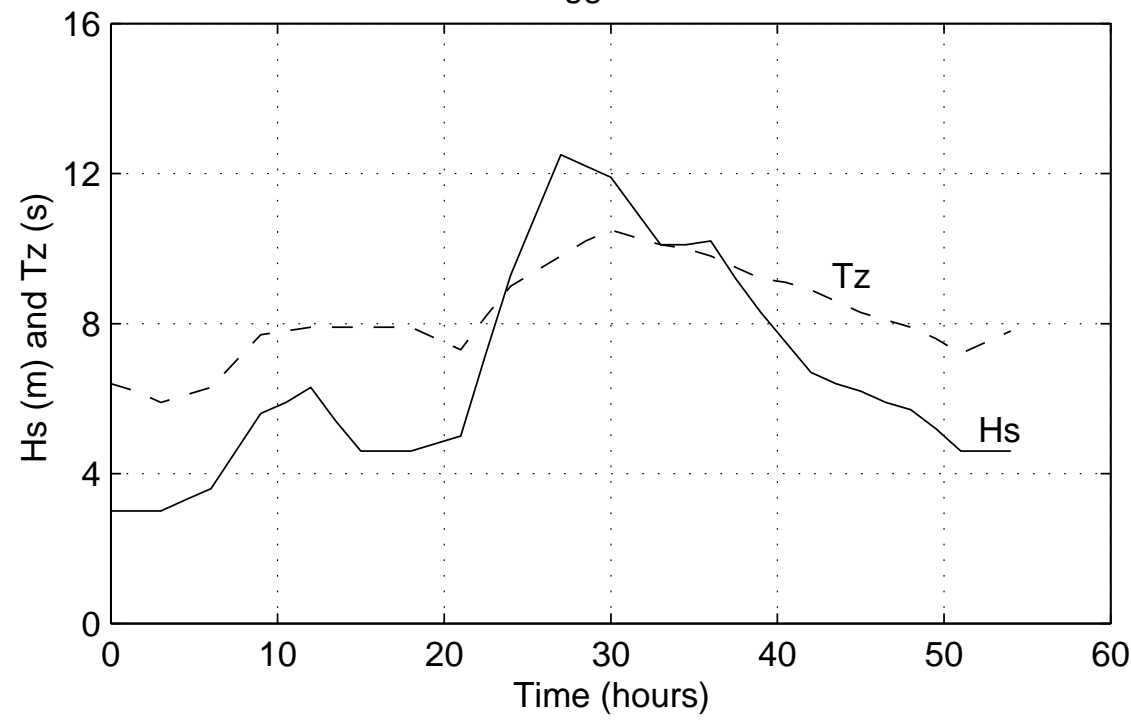

13. Frigg storm. Probability distribution for the maximum crest height (dotted: Gauss; solid: RS theory; dashed: Weibull model with $\alpha=2, \beta=2.45$ )

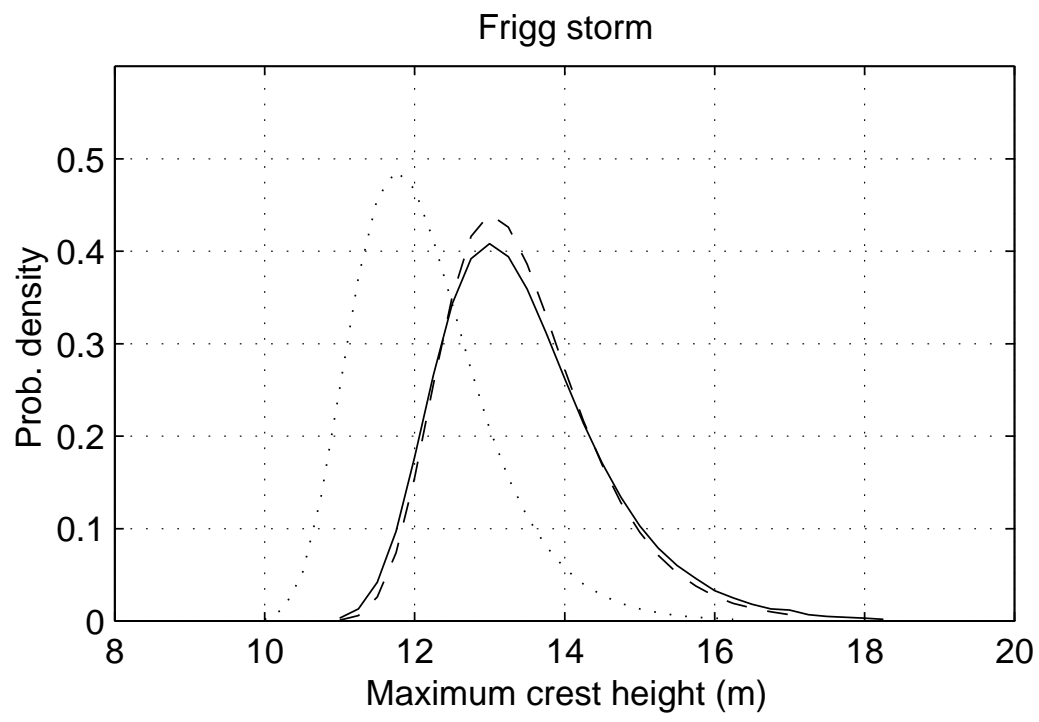


14. The Frigg storm. Probability distribution for the maximum wave height (solid: Forristall values; dashed: $\alpha=2$, $\beta=6.8$

Frigg storm

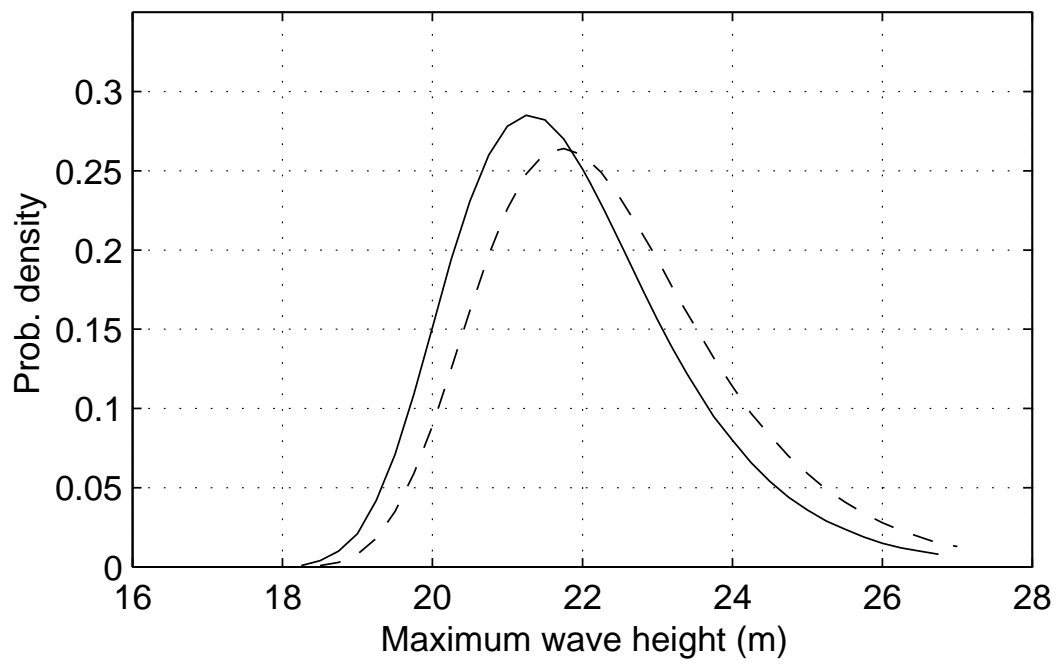

15. Probability distribution for the maximum crest height combined with the long term statistics mentioned in the text (dotted: Gauss; solid: RS theory; dashed: Weibull with $\alpha=2, \beta=2.45$ ).

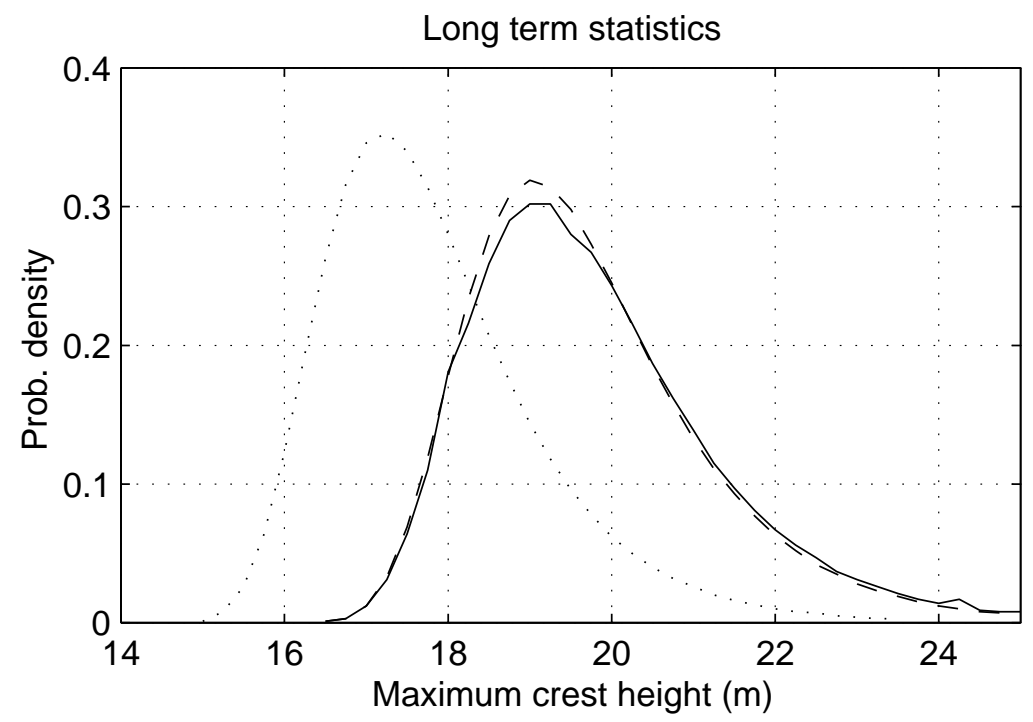


16. Probability distribution for the maximum wave height for the long term distribution of Hs (solid: Forristall values, dashed: $\alpha=2, \beta=6.8$ )

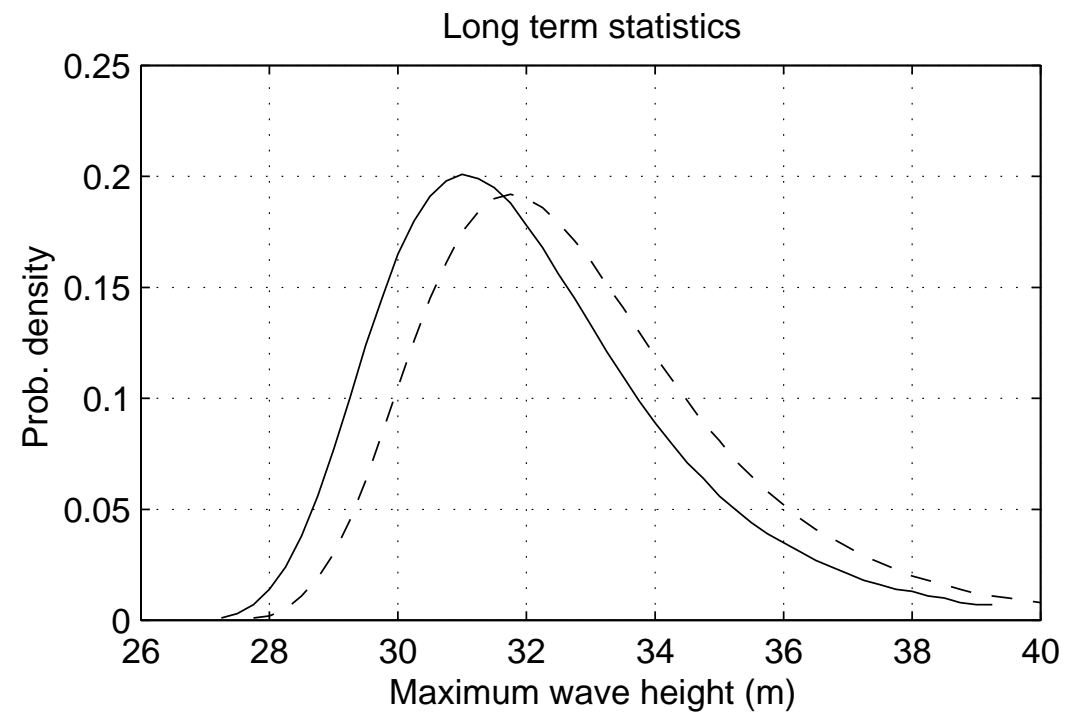




\section{Appendix 1 - Narrow-band non-linear transfer coefficients}

In the formulae below, $\kappa=k_{m} h$ is the dimensionless depth and $\kappa_{z}=k_{m} z$ the dimensionless vertical coordinate. Moreover,

$$
\begin{aligned}
& Q(\kappa)=\tanh \kappa+\kappa\left(1-(\tanh \kappa)^{2}\right) \quad Q(\infty)=1 \\
& c_{0}(\kappa)=2\left(c_{c s t}(\kappa)-c_{\text {diff }}(\kappa)\right)
\end{aligned}
$$

Expressions for vertical displacement, Eulerian (fixed point) measurements:

$$
\begin{aligned}
& c_{c s t}(\kappa)=0 \\
& c_{\text {diff }}(\kappa)=\frac{Q(\kappa)+\kappa\left(1-(\tanh \kappa)^{2}\right)}{Q(\kappa)^{2}-4 \kappa \tanh \kappa} \quad c_{\text {diff }}(\infty)=0 \\
& c_{\text {sum }}(\kappa)=\frac{1}{4}\left(\frac{2+\left(1-(\tanh \kappa)^{2}\right)}{(\tanh \kappa)^{3}}\right) \quad c_{\text {sum }}(\infty)=\frac{1}{2}
\end{aligned}
$$

Expressions for vertical displacement, Lagrangian (particle displacement) measurements:

$$
\begin{aligned}
& c_{\text {cst }}(\kappa)=\frac{1}{2} \frac{1}{\tanh \kappa} \quad c_{c s t}(\infty)=\frac{1}{2} \\
& c_{\text {diff }}(\kappa)=\frac{1}{2} \frac{1}{\tanh \kappa}\left(\frac{2(\tanh \kappa)^{2}(1-2 \kappa \tanh \kappa)+Q(\kappa)^{2}}{Q(\kappa)^{2}-4 \kappa \tanh \kappa}\right) \\
& c_{\text {sum }}(\kappa)=\frac{3}{4}\left(\frac{\left(1-(\tanh \kappa)^{2}\right)}{(\tanh \kappa)^{3}}\right) \quad c_{\text {diff }}(\infty)=\frac{1}{2}
\end{aligned}
$$

Expressions valid for both Eulerian and Lagrangian measurements:

$$
\begin{aligned}
& c_{\text {diff }}(\kappa)+c_{\text {sum }}(\kappa)=\frac{1}{4} \frac{1}{\tanh \kappa^{3}}\left(\frac{Q(\kappa)^{2}\left(3-(\tanh \kappa)^{2}\right)-12 \kappa \tanh \kappa\left(1-(\tanh \kappa)^{2}\right)+4(\tanh \kappa)^{4}(1-2 \tanh \kappa)}{Q(\kappa)^{2}-4 \kappa \tanh \kappa}\right) \\
& c_{0}(\kappa)=-\frac{Q(\kappa)+\kappa\left(1-(\tanh \kappa)^{2}\right)}{Q(\kappa)^{2}-4 \kappa \tanh \kappa} \quad c_{0}(\infty)=0
\end{aligned}
$$

Dynamic pressure $(\mathrm{P} /(\rho \mathrm{g}))$ :

$$
\begin{aligned}
& T=\frac{1+\left(\tanh \left(\kappa+\kappa_{z}\right)\right)^{2}}{1-\left(\tanh \left(\kappa+\kappa_{z}\right)\right)^{2}} \\
& c_{c s t}(\kappa)=-\frac{1}{4} \frac{1-(\tanh \kappa)^{2}}{\tanh \kappa} T \quad c_{c s t}(\infty)=-\frac{1}{2} \exp 2 \kappa_{z}
\end{aligned}
$$




$$
\begin{aligned}
& c_{\text {diff }}(\kappa)=-\frac{1}{4} \frac{1}{\tanh \kappa}\left(\left(1-(\tanh \kappa)^{2}\right) T-\frac{Q(\kappa)\left(1-(\tanh \kappa)^{2}\right)+4 \tanh \kappa}{Q(\kappa)^{2}-4 \kappa \tanh \kappa} Q(\kappa)\right) \\
& c_{\text {diff }}(\infty)=-\frac{1}{2} \exp 2 \kappa_{z} \\
& c_{\text {sum }}(\kappa)=-\frac{1}{4} \frac{1}{(\tanh \kappa)^{3}}\left((\tanh \kappa)^{2}-3 T\left(1-(\tanh \kappa)^{2}\right)\right)\left(1-(\tanh \kappa)^{2}\right) \quad c_{\text {sum }}(\infty)=0 \\
& c_{\text {diff }}(\kappa)+c_{\text {sum }}(\kappa)=-\left(\frac{1\left(1-(\tanh \kappa)^{2}\right)\left(4(\tanh \kappa)^{2}-3\right)}{4} T-\frac{2 \kappa\left(1-(\tanh \kappa)^{2}\right)+\tanh \kappa}{(\tanh \kappa)^{3}}\right)
\end{aligned}
$$

